\title{
Aging Reduces Estradiol Protection Against Neural but Not Metabolic Effects of Obesity in Female 3xTg-AD Mice
}

\author{
Amy Christensen, Jiahui Liu and Christian J. Pike* \\ Davis School of Gerontology, University of Southern California, Los Angeles, CA, United States
}

Vulnerability to Alzheimer's disease (AD) is increased by several risk factors, including midlife obesity, female sex, and the depletion of estrogens in women as a consequence of menopause. Conversely, estrogen-based hormone therapies have been linked with protection from age-related increases in adiposity and dementia risk, although treatment efficacy appears to be affected by the age of initiation. Potential interactions between obesity, $A D$, aging, and estrogen treatment are likely to have significant impact on optimizing the use of hormone therapies in postmenopausal women. In the current study, we compared how treatment with the primary estrogen, 17ß-estradiol (E2), affects levels of AD-like neuropathology, behavioral impairment, and other neural and systemic effects of preexisting diet-induced obesity in female 3xTg-AD mice. Importantly, experiments were conducted at chronological ages associated with both

OPEN ACCESS

Edited by:

P. Hemachandra Reddy,

Texas Tech University Health Sciences Center, United States

Reviewed by: Lei Yu,

Thomas Jefferson University,

United States Linda Ann Bean,

University of Florida, United States

*Correspondence: Christian J. Pike cjpike@usc.edu

Received: 04 February 2020 Accepted: 03 April 2020 Published: 05 May 2020

Citation:

Christensen A, Liu J and Pike CJ

(2020) Aging Reduces Estradiol

Protection Against Neural but Not Metabolic Effects of Obesity in Female $3 \times T g-A D$ Mice.

Front. Aging Neurosci. 12:113. doi: 10.3389/fnagi.2020.00113 the early and late stages of reproductive senescence. We observed that E2 treatment was generally associated with significantly improved metabolic outcomes, including reductions in body weight, adiposity, and leptin, across both age groups. Conversely, neural benefits of E2 in obese mice, including decreased $\beta$-amyloid burden, improved behavioral performance, and reduced microglial activation, were observed only in the early aging group. These results are consistent with the perspective that neural benefits of estrogen-based therapies require initiation of treatment during early rather than later phases of reproductive aging. Further, the discordance between E2 protection against systemic versus neural effects of obesity across age groups suggests that pathways other than general metabolic function, perhaps including reduced microglial activation, contribute to the mechanism(s) of the observed E2 actions. These findings reinforce the potential systemic and neural benefits of estrogen therapies against obesity, while also highlighting the critical role of aging as a mediator of estrogens' protective actions.

Keywords: aging, Alzheimer's disease, $\beta$-amyloid, estrogen, hormone therapy, microglia, obesity

\section{INTRODUCTION}

Alzheimer's disease $(\mathrm{AD})$ is a progressive neurodegenerative disorder that is the most common cause of dementia. While aging is the strongest risk factor for the disease, the development of $\mathrm{AD}$ is affected by numerous modifiable and non-modifiable risk factors (Hersi et al., 2017). One important and highly prevalent modifiable $\mathrm{AD}$ risk factor is obesity; people who are overweight or 
obese in midlife are at significantly increased risk for development of $\mathrm{AD}$ and related dementias in late life (Alford et al., 2018). Obesity-related conditions, including metabolic syndrome and type 2 diabetes, are also independently associated with increased dementia risk (Misiak et al., 2012; Bangen et al., 2019). Because there are no effective disease-modifying therapeutics for $\mathrm{AD}$, a promising interventional approach is to reduce vulnerability to $\mathrm{AD}$ by mitigating the effects of modifiable risk factors including obesity.

Another important $\mathrm{AD}$ risk factor is female sex. Women have a higher lifetime risk of $\mathrm{AD}$ than men as well as greater prevalence, with women accounting for approximately twothirds of AD cases (Mazure and Swendsen, 2016; Alzheimer's Association, 2018). There are numerous sex differences in the development, progression and manifestation of $\mathrm{AD}$ that likely contribute to the disease's female bias (Pike, 2017). One significant component is the depletion of sex steroid hormones, in particular $17 \beta$-estradiol (E2), which occurs as a consequence of menopause. Estradiol exerts numerous beneficial effects in tissues throughout the body, including the brain, such that the loss of estradiol can increase risks for dysfunction and disease (Clegg et al., 2017; Merlo et al., 2017). In transgenic mouse models of $\mathrm{AD}$, depletion of estrogens by ovariectomy accelerates the onset and progression of AD-like neuropathology and cognitive impairment, whereas treatment with E2 protects against these effects (Pike et al., 2009; Li and Singh, 2014). In women, early surgical menopause and other causes of reduced lifetime exposure to estrogens are associated with elevated AD risk (Bove et al., 2014; Pike, 2017; Jang et al., 2018). Whether estrogenbased hormone therapies (HT) significantly reduce AD risk in postmenopausal women is uncertain, with conflicting findings across studies (Yaffe et al., 1998; Hamoda et al., 2016; Lobo et al., 2016; Lobo, 2017). One hypothesis that appears to explain inconsistencies in the literature is that HT must be administered near the time of menopause in order to protect against dementia (Depypere et al., 2016; Lobo, 2017; Marjoribanks et al., 2018). Thus, reproductive and/or chronological age may be critical variables in the efficacy of HT as preventive strategy for AD.

Importantly, obesity, female sex, and reproductive and chronological aging are interactive AD risk factors. For example, estrogen depletion resulting from menopause is linked not only with heightened $\mathrm{AD}$ risk, but also increased adiposity and risk for metabolic dysfunction, which in turn are associated with elevated AD risk (Christensen and Pike, 2015). Estrogens can reduce adiposity and improve both metabolic symptoms (Brown et al., 2010) and neural functions (Frick et al., 2015) but are also predicted to exhibit reduced efficacy with increasing age (Morrison et al., 2006). In a recent investigation of these interactions in female $3 \mathrm{xTg}$ - $\mathrm{AD}$ mice, we found that E2-based $\mathrm{HT}$ initiated at the onset of diet-induced obesity reduced ADrelated pathology and cognitive impairment in early but not late middle age (Christensen and Pike, 2017). These findings are consistent with the conclusion that HT protects against the development of obesity-related neural impairment in $\mathrm{AD}$ mice, with the preventive outcomes exhibiting age-dependence. What remains unclear is whether estrogens can also protect against pre-existing obesity and thus function as a treatment for a modifiable risk factor of $\mathrm{AD}$. The current study examines this latter question. Female 3xTg-AD mice were maintained on an obesogenic diet for 2 months beginning in early middle age (early-MA) prior to the onset of reproductive aging or in late middle-age (late-MA) well beyond the cessation of ovarian cycling, then treated with estradiol for 2 months in the continued presence of obesogenic diet. The data demonstrate that estradiol reduces metabolic consequences of obesity across age groups, but significantly improves aspects of $\mathrm{AD}$-related pathology and cognitive impairment only when administered in early middle age.

\section{MATERIALS AND METHODS}

\section{Animals}

A colony of 3xTg-AD mice (Oddo et al., 2003) were maintained at the University of Southern California with ad libitum access to chow and water under a $12 \mathrm{~h} \mathrm{light/dark} \mathrm{schedule} \mathrm{(lights} \mathrm{on} \mathrm{at} \mathrm{6:00}$ $\mathrm{AM})$. Female $3 \mathrm{xTg}-\mathrm{AD}$ mice were randomized to experimental groups that were maintained on either control (10\% calories from fat and 7\% from sugar; catalog \#D12450Ji, Research Diets, Inc., New Brunswick, NJ, United States) or high-fat diet (60\% calories from fat and 7\% from sugar; catalog \#D12492i, Research Diets, Inc) for 16-weeks. Experimental groups were exposed to diet between ages 5-9 months ( $n=8$ /group) or 16-20 ( $n=6-$ 7 /group) months, chronological age spans in female mice that correspond to early stages of both middle age (early-MA) and reproductive senescence versus late stages of middle age (lateMA) and reproductive senescence, respectively (Nelson et al., 1982; Felicio et al., 1984; Finch, 2014). Animals were weighed weekly during the diet exposure period. After the first 8 weeks of diet, animals were anesthetized with the inhalant isoflurane (3\%), then implanted subcutaneously (between the shoulder blades) with a Silastic capsule (1.47 mm ID x $1.96 \mathrm{~mm}$ OD; Dow Corning, Midland, MI, United States). Each capsule had a total length of $7 \mathrm{~mm}$ with the inner $3 \mathrm{~mm}$ packed with cholesterol (vehicle) or a 1:3 mixture of E2 to cholesterol. In 5 monthsold, gonadally intact female mice maintained on control diet, E2 capsules yielded a statistically non-significant trend toward increased plasma E2 $(114.5 \pm 40.9 \mathrm{pg} / \mathrm{mL})$ relative to vehicletreated $(42.1 \pm 32.7 \mathrm{pg} / \mathrm{mL})$ mice $(n=4-5$, $t$-test: $p=0.23)$. Eight weeks following capsule implantation (16 weeks after initiation of diet), all animals were euthanized. The experimental design is summarized in Figure 1. Brains were immersion fixed in $4 \%$ paraformaldehyde for $72 \mathrm{~h}$ and subsequently stored at $4^{\circ} \mathrm{C}$. Plasma was collected and stored in aliquots at $-80^{\circ} \mathrm{C}$. Visceral and retroperitoneal fat pads were dissected, weighed, and stored at $-80^{\circ} \mathrm{C}$. All procedures were conducted in accordance with the guidelines set forth by the university's Institutional Animal Care and Use Committee and under the supervision of university veterinarians.

\section{Glucose Measurements}

Fasting glucose (16 h overnight fast) was measured at weeks 0,8 , and 15 of the experimental period (Figure 1). Five microliters of blood were collected on a glucose test strip and assayed using a 


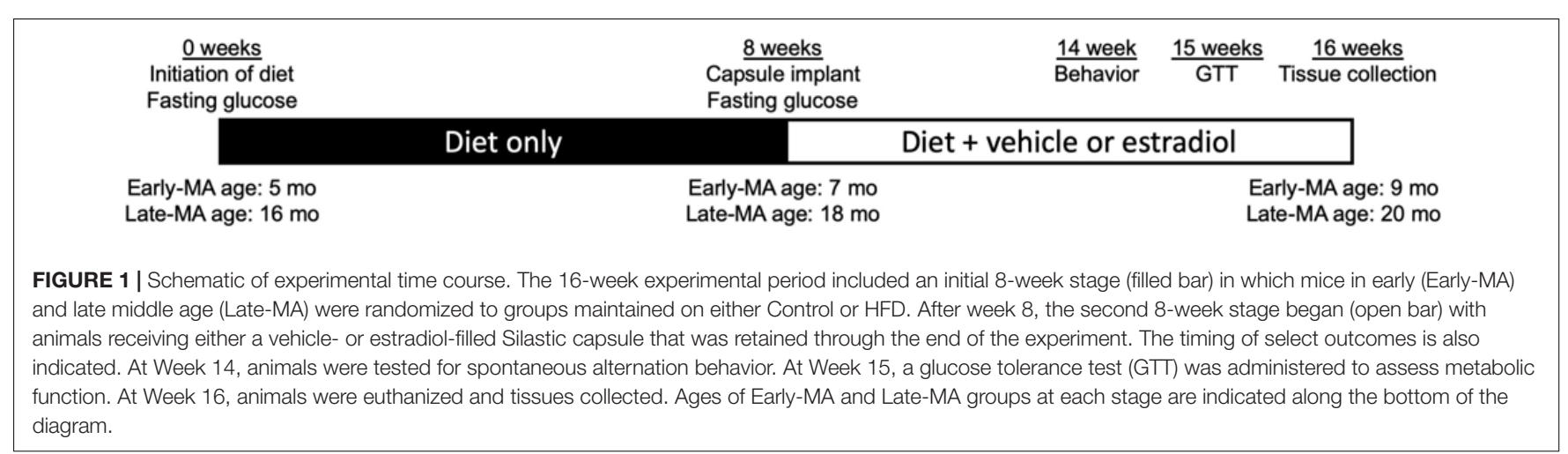

Precision Xtra glucose monitor (Abbott Laboratories). A glucose tolerance test was performed after 15 weeks of diet ( 7 weeks after the start of E2 or vehicle treatment). In brief, animals were orally gavaged with $2 \mathrm{~g} / \mathrm{kg}$ D-glucose and blood glucose levels were measured at $0,15,30,60$, and 120 min thereafter.

\section{Spontaneous Alternation Behavior}

At week 14, animals were behaviorally assessed using the spontaneous alternation behavior test in a Y-maze (Figure 1). The spontaneous alternation test is dependent upon the hippocampus and other limbic structures (Lalonde, 2002) and assesses spatial memory and attention toward novelty (Hughes, 2004). Animals were allowed to acclimate to the behavior room for $30 \mathrm{~min}$ prior to testing. Next, animals were placed in the long arm of a Y-maze facing away from the other arms to start the test. Arm entries (at least two paws placed into an arm) were recorded for $5 \mathrm{~min}$. Animals with fewer than 10 arm entries were excluded from analysis. Percent spontaneous alternation was calculated as number of correct triplicates divided by total number of triplicate arm entries.

\section{Immunohistochemistry}

Fixed hemibrains were sectioned exhaustively in the horizontal plane at $40 \mu \mathrm{m}$ using a vibratome (Leica Biosystems). Sections were stored singly in PBS with $0.03 \%$ sodium azide at $4^{\circ} \mathrm{C}$ until immunohistochemistry was performed. Every eighth section (from a total of $\sim 100$ per brain) was immunostained for $\beta$-amyloid $(\mathrm{A} \beta)$ as previously described (Christensen and Pike, 2017). In brief, tissue sections containing hippocampus were pretreated with $95 \%$ formic acid for $5 \mathrm{~min}$, then washed three times for $5 \mathrm{~min}$ in TBS, followed by a $10 \mathrm{~min}$ rinse with an endogenous peroxidase blocking solution. Next, sections were rinsed in TBS/ $0.1 \%$ Triton-X before being incubated for $30 \mathrm{~min}$ in a blocking solution consisting of TBS/2\% bovine serum albumin. Sections were incubated overnight at $4{ }^{\circ} \mathrm{C}$ with anti- $\mathrm{A} \beta$ antibody (1:300; Life Technologies; Cat \#71-5800) diluted in blocking solution. Immunostaining was also conducted in the absence of formic acid pretreatment with the following primary antibodies: doublecortin (1:2500; Santa Cruz) as a marker of new neurons, clone AT8 (1:750; Thermo) for phosphorylated tau (phosphotau), Iba-1 (1:2000; Wako) for microglia. Sections incubated in primary antibody were washed and then incubated with the appropriate secondary antibody (Vector Laboratories) for $1 \mathrm{~h}$ and processed for diaminobenzidene visualization using Vectastain ABC Elite kit (Vector Laboratories). Stained sections were airdried overnight, dehydrated in a series of graded alcohols, then coverslipped with Krystalon (EMD Millipore).

\section{$\beta$-Amyloid Load}

To assess $A \beta$ load, the percentage area of $A \beta$ immunoreactivity was determined, as previously described (Christensen and Pike, 2018). In brief, non-overlapping high magnification images were collected from the subiculum (three fields/section) and CA1 hippocampal subfield (three fields/section) across four tissue sections per brain, for a total of $\sim 24$ images per brain. Images were digitally captured using an Olympus BX50 microscope and DP74 camera paired with a computer running CellSens software (Olympus). Images were converted to grayscale and thresholded using NIH ImageJ $1.50 \mathrm{i}$ to yield binary images separating positive and negative immunostaining. A $\beta$ load was calculated as the percentage of the total pixels that was positively immunolabeled.

\section{Quantification of Immunolabeled Cells}

Doublecortin- and tau-immunoreactive cells were counted from 4 sections per brain. Positive labeling was defined as cells that were darkly stained across the majority of the soma. Doublecortin-labeled cells were counted across the entire dentate gyrus. Tau-positive cells were counted across the entire subiculum and CA1 regions of the hippocampus. Microglial activation was based on morphological analysis of Iba-1 immunoreactive cells, as previously described (Christensen and Pike, 2017, 2018). Density of Iba-1 immunoreactive cells in the hippocampus was estimated by two-dimensional counts. Briefly, an Olympus BX50 microscope equipped with a motorized stage and computer-guided CASTGrid software (Olympus) was used for unbiased sampling. In four sections per animal, the area containing the subiculum and CA1-CA3 subregions of the hippocampus (excluding the dentate gyrus) was sampled at high magnification. Within each field, cells within a counting frame $\left(3000 \mu \mathrm{m}^{2}\right)$ were used for analysis. Microglia were classified as either type 1, (many thin, ramified processes), type 2 (short, thick processes and a rod-shaped cell body), or type 3 (no or few short non-ramified processes or many filapodial processes) cells. Type 2 and 3 cells were considered to exhibit an activated microglia morphological phenotype. 


\section{ELISAs}

Estradiol concentration in plasma was measured using an estradiol ELISA (Calbiotech) according to the manufacturer's instructions. Plasma leptin was measured using a leptin ELISA (Millipore), according to the manufacturer's protocol.

\section{PCR}

For RNA extractions, visceral fat pads were dissociated using a dounce homogenizer in TRIzol reagent (Invitrogen), following the manufacturer's protocol except that a $10 \mathrm{~min} 12000 \times \mathrm{g}$ spin was added prior to the addition of chloroform to reduce lipid content. The resultant RNA pellet was treated with RNase-free DNase I (Epicentre) for $30 \mathrm{~min}$ at $37^{\circ} \mathrm{C}$, and a phenol/chloroform extraction was performed to isolate RNA. The iScript cDNA synthesis system (Bio-Rad) was used to reverse transcribe cDNA from $1 \mu \mathrm{g}$ of purified RNA. Real-time quantitative PCR was performed on the resulting cDNA using ssoAdvanced Universal SYBR Green Supermix (Bio-Rad) and a Bio-Rad CFX96 Touch Real-Time PCR Detection System. As a general rule, samples from all experimental groups were run on each plate and $\Delta \Delta \mathrm{Ct}$ results were compared to relative to the early middle age control mice (control diet + vehicle capsule). PCR was performed using the following primer pairs: CD68 (forward: TTCTGCTGTGGAAATGCAAG and reverse: AGAGG GGCTGGTAGGTTGAT) F4/80 (forward: TGCATCTAGCAA TGGACAGC and reverse: GCCTTCTGGATCCATTTGAA), interleukin-6 (IL-6; forward: AGTTGCCTTCTTGGGACTGA and reverse: TCCACGATTTCCCAGAGAAC), succinate dehydrogenase complex, subunit A (SDHA; forward: ACACA GACCTGGTGGAGACC and reverse: GGATGGGCTTGGAGT AATCA) and hypoxanthine guanine phosphoribosyltransferase (HPRT; forward: AAGCTTGCTGGTGAAAAGGA and reverse: TTGCGCTCATCTTAGGCTTT). Expression levels of CD68, F4/80, and IL-6 were normalized to the average expression of the two reference markers, SDHA and HPRT.

\section{Statistics}

All data are reported as the mean \pm the standard error of the mean. Data were analyzed using GraphPad Prism version 8. Most data were statistically analyzed using two-way ANOVA followed by Tukey's post hoc tests when appropriate. Three-way repeated measure ANOVA followed by Tukey's post hoc tests was used to analyze data measured over time (body weight, fasting glucose, GTT) and to compare the effects of age, diet and hormone treatment. Statistical analyses are listed in Tables 1, 2.

\section{RESULTS}

\section{Estradiol Affects Body Mass and Adiposity in Both Early and Late Middle Age}

We sought to determine how the protective efficacy of E2 is affected by preexisting obesity across the transitions associated with middle age, including reproductive senescence. To accomplish this, female $3 \times \mathrm{Tg}$-AD mice were implanted with a Silastic capsule containing either vehicle (Veh) or E2 halfway through a 16-week exposure to either a control (Con) or high-fat (HFD) diet during ages corresponding to early (early-MA) or late (late-MA) middle age (Figure 1).

By week 8 of the experimental period, HFD was associated with significantly increased body weight relative to Con in both the early-MA and late-MA groups [Figure $\mathbf{2 A}$, $F_{\text {diet }}(1,28)=41.4, p<0.0001 ; 2 \mathrm{~B}, F_{\text {diet }}(1,28)=54.5, p<0.0001$; $2 \mathrm{~F}, F_{\text {diet }}(1,23)=35.1, p<0.0001$; and $2 \mathrm{G}, F_{\text {diet }}(1,23)=52.5$, $p<0.0001$ ] (Table 1). In the early-MA groups, vehicletreated mice on HFD continued to show significant weight gain throughout the next 8 weeks, whereas E2-treated mice exhibited no further increase in body weight although they remained significantly heavier than both groups of mice maintained on Con diet (Figure 2A). There was a significant main effect of hormone treatment and a significant interaction between diet and hormone treatment on percentage of weight change after the capsule implant [Figure 2C, $F_{\text {hormone }}(1,28)=24.7$, $\left.p<0.0001 ; F_{\text {interaction }}(1,28)=25.7, p<0.0001\right]$. Interestingly, unlike the early-MA mice, the late-MA mice on HFD lost weight after implantation with E2 (Figure 2F). There were significant main effects of both diet and hormone treatment as well as an interaction between these two factors in the percent weight change in the late-MA animals [Figure $2 \mathbf{H}, F_{\text {diet }}(1,23)=5.5$, $p<0.05 ; F_{\text {hormone }}(1,23)=5.4, p<0.05 ; F_{\text {interaction }}(1,23)=9.3$, $p<0.001]$. There was no significant weight change difference after hormone treatment in animals of either reproductive age that received the Con diet (Table 1).

The effects of diet and hormone treatments on body weight were paralleled by differences across groups in the weights of fat depots. Within each age group, retroperitoneal (early-MA, Figure 2D and late-MA, Figure 2I) and visceral (early-MA, Figure 2E and late-MA, Figure 2J) fat pad weights (normalized to body weight) showed significant main effects of diet and hormone as well as an interaction between the two treatments (see Table 1 for statistical values). Specifically, HFD was associated with increased fat pad weight whereas E2 attenuated the HFDinduced increases [early-MA, Figure $2 D, F_{\text {interaction }}(1,28)=10.7$, $p<0.01$; Figure 2E, $F_{\text {interaction }}(1,28)=21.1, p<0.0001$; Figure 2I, $F_{\text {interaction }}(1,23)=6.4, p<0.05$; and late-MA Figure 2J, $F_{\text {interaction }}(1,23)=5.9, p<0.05$ ].

Data analyses were designed to determine the independent and interactive effects of HFD and E2 treatment in both early-MA and late-MA female mice. Although not a primary objective, the collected data can also be statistically analyzed to compare outcomes as a function of age (for complete statistical analysis, see Table 2). The initial body weights prior to experimental enrollment revealed that the late-MA animals started the experiment significantly heavier than the early-MA animals (Figure 2A vs. Figure 2F, $t$-test: $p<0.0001$ ). Further, there was a significant main effect of age on weight gain in both the initial and final 8 weeks of the experiment [Figure $2 \mathbf{B}$ vs. Figure 2G, $F_{\text {age }} \times$ diet $(1,26)=4.4, p<0.05$; and Figure 2C vs. Figure $\left.2 \mathbf{H}, F_{\text {age }} \times \operatorname{diet}(1,26)=24.6, p<0.0001\right]$. The late-MA animals showed lower percent weight gain on both Con diet and HFD than the early-MA mice [Figures 2A vs. Figure $2 F, F_{\text {time }} \times$ age $\times$ diet $\left.(4,104)=55.8, p<0.0001\right]$. However, 
TABLE 1 | Statistical analyses of data within age groups.

\begin{tabular}{|c|c|c|}
\hline Figure & Main Effects, Interactions & Post hoc comparisons \\
\hline $2 \mathrm{~A}$ & $\begin{array}{l}F_{\text {time }}(1.80,50.3)=76.3, p<0.0001 \\
F_{\text {diet }}(1,28)=41.4, p<0.0001 \\
F_{\text {time }} \times \operatorname{diet}(4,112)=41.4, p<0.0001 \\
F_{\text {time }} \times \text { hormone }(4,112)=8.11, p<0.0001 \\
F_{\text {time }} \times \text { diet } \times \text { hormone }(4,112)=9.25, p<0.0001\end{array}$ & $\begin{array}{r}8 \text { weeks: Con + Veh vs. HFD + Veh, } p=0.08 \\
\text { Con + E2 vs. HFD + E2, } p=0.08 \\
12 \text { weeks: Con + Veh vs. HFD + Veh, } p<0.05 \\
\text { Con + E2 vs. HFD + E2, } p=0.23 \\
16 \text { weeks: Con + Veh vs. HFD + Veh, } p<0.01 \\
\text { Con + E2 vs. HFD + E2, } p=0.20\end{array}$ \\
\hline $2 \mathrm{~B}$ & $F_{\text {diet }}(1,28)=54.5, p<0.0001$ & $\begin{array}{l}\text { Con + Veh vs. HFD + Veh, } p<0.0001 \\
\text { Con }+ \text { E2 vs. HFD + E2, } p<0.0001\end{array}$ \\
\hline $2 \mathrm{C}$ & $\begin{array}{l}F_{\text {hormone }}(1,28)=24.7, p<0.0001 \\
F_{\text {interaction }}(1,28)=25.7, p<0.0001\end{array}$ & $\begin{array}{l}\text { Con + Veh vs. HFD + Veh, } p=0.001 \\
\text { Con + E2 vs. HFD + E2, } p<0.05 \\
\text { HFD + Veh vs. HFD + E2, } p<0.0001\end{array}$ \\
\hline $2 \mathrm{D}$ & $\begin{array}{l}F_{\text {diet }}(1,28)=61.5, p<0.0001 \\
F_{\text {hormone }}(1,28)=20.7, p<0.0001 \\
F_{\text {interaction }}(1,28)=10.7, p<0.01\end{array}$ & $\begin{array}{l}\text { Con + Veh vs. HFD + Veh, } p<0.0001 \\
\text { Con + E2 vs. HFD + E2, } p<0.05 \\
\text { HFD + Veh vs. HFD + E2, } p<0.0001\end{array}$ \\
\hline $2 \mathrm{E}$ & $\begin{array}{l}F_{\text {diet }}(1,28)=62.5, p<0.0001 \\
F_{\text {hormone }}(1,28)=29.8, p<0.0001 \\
F_{\text {interaction }}(1,28)=21.1, p<0.0001\end{array}$ & $\begin{array}{l}\text { Con + Veh vs. HFD + Veh, } p<0.0001 \\
\text { Con + E2 vs. HFD + E2, } p<0.0001 \\
\text { HFD + Veh vs. HFD + E2, } p<0.0001\end{array}$ \\
\hline $2 \mathrm{~F}$ & $\begin{array}{l}F_{\text {time }}(2.49,57.3)=6.53, p<0.01 \\
F_{\text {diet }}(1,23)=35.1, p<0.0001 \\
F_{\text {hormone }}(1,23)=4.13, p=0.054 \\
F_{\text {time }} \times \operatorname{diet}(4,92)=23.8, p<0.0001 \\
F_{\text {time }} \times \text { hormone }(4,92)=9.7, p<0.0001 \\
F_{\text {time }} \times \text { diet } \times \text { hormone }(4,92)=10.6, p<0.0001\end{array}$ & $\begin{array}{r}8 \text { weeks: Con + Veh vs. HFD + Veh, } p<0.05 \\
\text { Con + E2 vs. HFD + E2, } p=0.07 \\
12 \text { weeks: Con + Veh vs. HFD + Veh, } p=0.056 \\
\text { Con + E2 vs. HFD + E2, } p=0.22 \\
16 \text { weeks: Con + Veh vs. HFD + Veh, } p=0.06 \\
\text { Con + E2 vs. HFD + E2, } p=0.07\end{array}$ \\
\hline $2 \mathrm{G}$ & $F_{\text {diet }}(1,23)=52.5, p<0.0001$ & $\begin{array}{l}\text { Con }+ \text { Veh vs. HFD }+ \text { Veh, } p<0.0001 \\
\text { Con }+ \text { E2 vs. HFD }+ \text { E2, } p<0.001\end{array}$ \\
\hline $2 \mathrm{H}$ & $\begin{array}{l}F_{\text {diet }}(1,23)=5.5, p<0.05 \\
F_{\text {hormone }}(1,23)=5.4, p<0.05 \\
F_{\text {interaction }}(1,23)=9.3, p<0.001\end{array}$ & $\begin{array}{l}\text { Con + E2 vs. HFD + E2, } p<0.01 \\
\text { HFD + Veh vs. HFD + E2, } p<0.01\end{array}$ \\
\hline $2 \mathrm{~J}$ & $\begin{array}{l}F_{\text {diet }}(1,23)=13.2, p<0.01 \\
F_{\text {hormone }}(1,23)=20.9, p<0.0001 \\
F_{\text {interaction }}(1,23)=5.9, p<0.05\end{array}$ & $\begin{array}{l}\text { Con + Veh vs. HFD + Veh, } p<0.01 \\
\text { HFD + Veh vs. HFD + E2, } p<0.001\end{array}$ \\
\hline 21 & $\begin{array}{l}F_{\text {diet }}(1,23)=15.1, p<0.001 \\
F_{\text {hormone }}(1,23)=18.9, p<0.001 \\
F_{\text {interaction }}(1,23)=6.4, p<0.05\end{array}$ & $\begin{array}{l}\text { Con + Veh vs. HFD + Veh, } p<0.001 \\
\text { HFD + Veh vs. HFD + E2, } p<0.001\end{array}$ \\
\hline $3 \mathrm{~A}$ & $\begin{array}{l}F_{\text {time }}(1.86,52.1)=11.6, p<0.0001 \\
F_{\text {diet }}(1,28)=3.74, p=0.06 \\
F_{\text {time }} \times \operatorname{diet}(2,56)=5.11, p<0.01\end{array}$ & No significant differences \\
\hline 3B & $\begin{array}{l}F_{\text {time }}(2.73,76.3)=137, p<0.0001 \\
F_{\text {diet }}(1,28)=6.53, p<0.05 \\
F_{\text {time }} \times \operatorname{diet}(4,112)=4.13, p<0.01\end{array}$ & No significant differences \\
\hline $3 \mathrm{C}$ & $\begin{array}{l}F_{\text {diet }}(1,28)=25.3, p<0.0001 \\
F_{\text {hormone }}(1,28)=11.0, p<0.01 \\
F_{\text {interaction }}(1,28)=8.1, p<0.01\end{array}$ & $\begin{array}{l}\text { Con + Veh vs. HFD + Veh, } p<0.0001 \\
\text { HFD + Veh vs. HFD + E2, } p<0.001\end{array}$ \\
\hline 3D & $F_{\text {diet }}(1,28)=8.0, p<0.01$ & No significant differences \\
\hline $3 \mathrm{E}$ & $\begin{array}{l}F_{\text {time }}(1.98,45.6)=8.6, p<0.001 \\
F_{\text {diet }}(1,23)=6.5, p<0.05\end{array}$ & No significant differences \\
\hline $3 F$ & $\begin{array}{l}F_{\text {time }}(2.85,57.0)=194, p<0.0001 \\
F_{\text {time }} \times \operatorname{diet}(4,80)=4.38, p<0.01 \\
F_{\text {diet }} \times \text { hormone }(1,20)=11.0, p<0.01 \\
F_{\text {time }} \times \text { diet } \times \text { hormone }(4,80)=3.15, p<0.05\end{array}$ & 60 min: Con + Veh vs. HFD + Veh, $p<0.001$ \\
\hline $3 G$ & $\begin{array}{l}F_{\text {diet }}(1,23)=9.5, p<0.01 \\
F_{\text {hormone }}(1,23)=14.0, p<0.01 \\
F_{\text {interaction }}(1,23)=6.8, p<0.05\end{array}$ & $\begin{array}{l}\text { Con + Veh vs. HFD + Veh, } p<0.01 \\
\text { HFD + Veh vs. HFD + E2, } p<0.001\end{array}$ \\
\hline $3 \mathrm{H}$ & $F_{\text {interaction }}(1,20)=16.1, p<0.001$ & $\begin{array}{l}\text { Con + Veh vs. HFD + Veh, } p<0.05 \\
\text { Con + Veh vs. Con + E2, } p<0.05\end{array}$ \\
\hline $4 \mathrm{~A}$ & $F_{\text {hormone }}(1,22)=12.9, p<0.01$ & HFD + Veh vs. HFD + E2, $p<0.05$ \\
\hline $4 \mathrm{~B}$ & No significant main effects & \\
\hline $4 \mathrm{C}$ & No significant main effects & \\
\hline $4 \mathrm{D}$ & No significant main effects & \\
\hline
\end{tabular}


TABLE 1 | Continued

\begin{tabular}{|c|c|c|}
\hline Figure & Main Effects, Interactions & Post hoc comparisons \\
\hline $5 \mathrm{E}$ & $F_{\text {interaction }}(1,28)=9.9, p<0.01$ & HFD + Veh vs. HFD + E2, $p<0.05$ \\
\hline $5 F$ & $\begin{array}{l}F_{\text {hormone }}(1,28)=3.1, p=0.09 \\
{\left[\text { without outlier: } F_{\text {hormone }}(1,27)=3.4, p=0.07\right]}\end{array}$ & \\
\hline $5 \mathrm{~K}$ & No significant main effects & \\
\hline $5 \mathrm{~L}$ & $F_{\text {diet }}(1,23)=6.3, p<0.05$ & Con + E2 vs. HFD + E2, $p<0.05$ \\
\hline $6 \mathrm{~A}$ & No significant main effects & \\
\hline $6 \mathrm{~B}$ & $\begin{array}{l}F_{\text {hormone }}(1,28)=16.6, p<0.001 \\
F_{\text {interaction }}(1,28)=4.2, p<0.05\end{array}$ & HFD + Veh vs. HFD + E2, $p<0.001$ \\
\hline $6 \mathrm{C}$ & $F_{\text {diet }}(1,22)=4.8, p<0.05$ & No significant differences \\
\hline $6 \mathrm{D}$ & $F_{\text {diet }}(1,22)=6.0, p<0.05$ & No significant differences \\
\hline $7 \mathrm{~A}$ & $F_{\text {diet }}(1,28)=7.1, p<0.05$ & Con + Veh vs. HFD + Veh, $p<0.05$ \\
\hline $7 \mathrm{~B}$ & $F_{\text {diet }}(1,28)=7.5, p<0.05$ & Con + Veh vs. HFD + Veh, $p<0.05$ \\
\hline $7 \mathrm{C}$ & $F_{\text {diet }}(1,28)=4.6, p<0.05$ & No significant differences \\
\hline $7 \mathrm{D}$ & $F_{\text {diet }}(1,23)=9.8, p<0.01$ & Con + Veh vs. HFD + Veh, $p<0.05$ \\
\hline $7 \mathrm{E}$ & $F_{\text {diet }}(1,23)=4.4, p<0.05$ & No significant differences \\
\hline $7 F$ & $F_{\text {hormone }}(1,23)=5.2, p<0.05$ & No significant differences \\
\hline
\end{tabular}

net increases in body mass (measured in grams) were similar, suggesting differences in percent weight gain largely reflect the differences in initial weights. Although both groups gained approximately the same total grams from 0 to 8 weeks, only the early-MA females on HFD continued to gain weight during weeks 8-16. Also, there was a significant effect of age on the response to $\mathrm{E} 2$, with late-MA animals losing significantly more weight than early-MA animals on HFD [Figure 2A vs. Figure 2F, $F_{\text {time }} \times$ age $\times$ hormone $\left.(4,104)=55.7, p<0.0001\right]$. There were no significant differences between the ages in the normalized weights of the fat pads (Figure $2 \mathrm{D}$ vs. Figure 2I and Figure $2 \mathrm{E}$ vs. Figure 2J).

\section{Estradiol Affects Metabolic Measures in Both Early and Late Middle Age}

Plasma levels of leptin determined at week 16 closely followed changes in adiposity. In both age groups, fasting leptin concentrations were significantly higher in HFD groups with $\sim 8$ fold increase in the early-MA group and $\sim 5$-fold elevation in the late-MA group [Figure 3C, $F_{\text {diet }}(1,28)=25.3, p<0.0001$; and $\left.3 G, F_{\text {diet }}(1,23)=9.5, p<0.01\right]$. Further, E2 treatment robustly inhibited the HFD-associated increase in leptin levels. In early-MA, E2 was associated with $\sim 70 \%$ decrease in leptin relative to the vehicle-treated HFD group, whereas in late-MA the E2-induced decrease in leptin was $\sim 85 \%$ [Figure 3C, $F_{\text {interaction }}(1,28)=8.1, p<0.01$; and Figure 3G, $\left.F_{\text {interaction }}(1,23)=6.8, p<0.05\right]$ (Table 1).

To determine whether the observed HFD-induced increases in body weight, fat mass, and leptin levels were associated with functional metabolic impairments, we assessed both fasting glucose levels and glucose tolerance. In general, HFD diet was linked with increases in fasting glucose levels, which were measured at weeks 0,8 , and 15 . There was a non-significant trend [Figure 3A, $F_{\text {diet }}(1,28)=3.74, p=0.06$ ] of a main effect of diet and a significant interaction between time and diet on fasting glucose in the early-MA mice [Figure $\mathbf{3 A}, F_{\text {time }} \times \operatorname{diet}(2,56)=5.11$, $p<0.01]$. In the late-MA mice, there was a significant main effect of diet on fasting glucose [Figure $3 \mathrm{E}, F_{\text {diet }}(1,23)=6.5, p<0.05$ ] Similarly, HFD was associated with impairments in glucose tolerance. There was a significant main effect of diet on glucose tolerance in the early-MA animals [Figure 3B, $F_{\text {diet }}(1,28)=6.53$, $p<0.05$ ] that was also reflected in the area under the curve measurements [Figure 3D, $F_{\text {diet }}(1,28)=8.0, p<0.01$ ]. In the late-MA mice, HFD resulted in poorer glucose tolerance that was significantly different from Con at the $60 \mathrm{~min}$ time point [Figure 3F, $F_{\text {time }} \times$ diet $\times$ hormone $(4,80)=3.15, p<0.05$ ]. Further, there were significant interactive effects between diet and hormone treatment on the area under the curve [Figure $3 \mathbf{H}$, $\left.F_{\text {interaction }}(1,20)=16.1, p<0.001\right]$ such that HFD significantly impaired glucose tolerance specifically in vehicle-treated mice $(p<0.05)$ and E2 worsened glucose tolerance in the Con $\operatorname{diet}(p<0.05)$.

We also considered the independent effects of age on metabolic outcomes. In general, metabolic consequences of HFD and E2 treatments were similar with HFD generally leading to a poorer metabolic profile regardless of chronological age and E2 improving some of the metabolic changes induced by HFD, including fasting glucose. However, there was a non-significant trend for an age $\times$ diet interaction in the GTT AUC with late-MA animals in all groups appearing to have a similar or greater AUC compared to the early-MA animals [Figure 3D vs. Figure $\mathbf{3 H}$, $\left.F_{\text {age } \times \operatorname{diet}}(1,24)=3.5, p=0.08\right]$.

\section{Behavioral Performance Is Improved by Estradiol Treatment Only in Early-MA Mice}

HFD in mice has been associated with impairments (Winocur and Greenwood, 2005; Park et al., 2010; McLean et al., 2018) and E2 with improvements (Carroll et al., 2007; Barker and Galea, 2008; Christensen and Pike, 2017) in both hippocampaldependent cognitive tasks and hippocampal neurogenesis. 
TABLE 2 | Statistical analyses of data between age groups.

\begin{tabular}{|c|c|c|}
\hline Figure & Main effects, Interactions & Post hoc comparisons \\
\hline $2 \mathrm{~A}$ and $2 \mathrm{~F}$ & $\begin{array}{l}F_{\text {time }} \times \text { age } \times \operatorname{diet}(4,104)=55.8, p<0.0001 \\
F_{\text {time }} \times \text { age } \times \text { hormone }(4,104)=55.7, p<0.0001\end{array}$ & $\begin{array}{l}0 \text { weeks: E-MA Con + Veh vs. L-MA Con + Veh, } p<0.001 \\
\text { E-MA HFD + Veh vs. L-MA HFD + Veh, } p<0.001 \\
\text { E-MA HFD + E2 vs. L-MA HFD + E2, } p<0.05 \\
4 \text { weeks: E-MA HFD + Veh vs. L-MA HFD + Veh, } p<0.0001 \\
\text { E-MA HFD + E2 vs. L-MA HFD + E2, } p<0.01 \\
8 \text { weeks: E-MA HFD + Veh vs. L-MA HFD + Veh, } p<0.0001 \\
\text { E-MA HFD + E2 vs. L-MA HFD + E2, } p<0.05 \\
12 \text { weeks: E-MA HFD + Veh vs. L-MA HFD + Veh, } p<0.01 \\
16 \text { weeks: E-MA HFD + Veh vs. L-MA HFD + Veh, } p<0.05\end{array}$ \\
\hline $2 \mathrm{~B}$ and $2 \mathrm{G}$ & $F_{\text {age }} \times \operatorname{diet}(1,26)=4.4, p<0.05$ & No significant differences \\
\hline $2 \mathrm{C}$ and $2 \mathrm{H}$ & $\begin{array}{l}F_{\text {age }} \times \operatorname{diet}(1,26)=24.6, p<0.0001 \\
F_{\text {age }} \times \text { hormone }(1,27)=29.7, p<0.0001\end{array}$ & $\begin{array}{l}\text { E-MA HFD + Veh vs. L-MA HFD + Veh, } p<0.001 \\
\text { E-MA HFD + E2 vs. L-MA HFD + E2, } p<0.05\end{array}$ \\
\hline
\end{tabular}

\begin{tabular}{|c|c|}
\hline 2D and $2 \mathrm{I}$ & No significant main effects \\
\hline $2 \mathrm{E}$ and $2 \mathrm{~J}$ & No significant main effects \\
\hline $3 \mathrm{~A}$ and $3 \mathrm{E}$ & 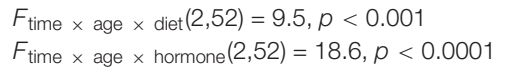 \\
\hline 3B and $3 F$ & $\begin{array}{l}F_{\text {time }} \times \text { age } \times \text { diet }(4,96)=174.5, p<0.0001 \\
F_{\text {time }} \times \text { age } \times \text { hormone }(4,96)=151.0, p<0.0001\end{array}$ \\
\hline $3 C$ and $3 G$ & No significant main effects \\
\hline $3 \mathrm{D}$ and $3 \mathrm{H}$ & $F_{\text {age }} \times \operatorname{diet}(1,24)=3.5, p=0.08$ \\
\hline $4 \mathrm{~A}$ and $4 \mathrm{C}$ & No significant main effects \\
\hline 4B and 4D & $F_{\text {age }} \times \operatorname{diet}(1,26)=28.5, p<0.0001$ \\
\hline $5 \mathrm{E}$ and $5 \mathrm{~K}$ & $F_{\text {age }} \times \operatorname{diet}(1,26)=57.2, p<0.0001$ \\
\hline $5 F a n d 5 L$ & $F_{\text {age }} \times \operatorname{diet}(1,26)=94.4, p<0.0001$ \\
\hline $6 \mathrm{~A}$ and $6 \mathrm{C}$ & $\begin{array}{l}F_{\text {age }} \times \operatorname{diet}(1,25)=28.0, p<0.0001 \\
F_{\text {age }} \times \operatorname{\text {hormone}}(1,26)=69.6, p<0.0001\end{array}$ \\
\hline $6 \mathrm{~B}$ and $6 \mathrm{D}$ & $\begin{array}{l}F_{\text {age }} \times \operatorname{diet}(1,25)=44.6, p<0.0001 \\
F_{\text {age }} \times \text { hormone }(1,26)=141.3, p<0.0001\end{array}$ \\
\hline 7A and 7D & $\begin{array}{l}F_{\text {diet }}(1,51)=17.3, p<0.0001 \\
F_{\text {diet }} \times \text { hormone }(1,51)=5.4, p<0.05\end{array}$ \\
\hline $7 \mathrm{~B}$ and $7 \mathrm{E}$ & $\begin{array}{l}F_{\text {diet }}(1,51)=11.1, p<0.01 \\
F_{\text {diet }} \times \text { hormone }(1,51)=4.4, p<0.05\end{array}$ \\
\hline $7 \mathrm{C}$ and $7 \mathrm{~F}$ & $\begin{array}{l}F_{\text {diet }}(1,51)=3.1, p=0.09 \\
F_{\text {age }} \times \operatorname{diet}(1,51)=3.3, p=0.08\end{array}$ \\
\hline
\end{tabular}

\author{
No significant differences \\ No significant differences
}

No significant differences

E-MA Control + Veh vs. L-MA Control + Veh, $p<0.001$ E-MA HFD + Veh vs. L-MA HFD + Veh, $p<0.05$

E-MA Control + Veh vs. L-MA Control + Veh, $p<0.001$

E-MA HFD + Veh vs. L-MA HFD + Veh, $p<0.0001$

E-MA Control + Veh vs. L-MA Control + Veh, $p<0.0001$

E-MA HFD + Veh vs. L-MA HFD + Veh, $p<0.0001$

E-MA Control + Veh vs. L-MA Control + Veh, $p<0.05$

E-MA HFD + Veh vs. L-MA HFD + Veh, $p<0.01$

E-MA HFD + E2 vs. L-MA HFD + E2, $p<0.0001$

E-MA Control + Veh vs. L-MA Control + Veh, $p<0.0001$

E-MA HFD + Veh vs. L-MA HFD + Veh, $p<0.01$

E-MA HFD + E2 vs. L-MA HFD + E2, $p<0.0001$

No significant differences

No significant differences

No significant differences

E-MA, early middle age; L-MA, late middle age.

To assess these outcomes, hippocampal-dependent cognitive function was assessed by spontaneous alternation behavior (SAB) and neurogenesis by levels of doublecortin (DCX)immunoreactive cells in the dentate gyrus. In early-MA females, there was a statistically non-significant trend toward reduced $\mathrm{SAB}$ performance by $\mathrm{HFD}$ and a significant main effect of hormone treatment such that E2 was associated with behavioral improvement [Figure 4A, $F_{\text {hormone }}(1,22)=12.9$, $p<0.01]$. Indeed, early-MA mice maintained on HFD and treated with E2 showed the greatest improvement with significantly better SAB performance than the HFD vehicletreated animals $(p<0.05)$. These effects appear to be unrelated to neurogenesis as there were no significant differences in numbers of DCX-immunoreactive cells across early-MA groups (Figure 4B). In late-MA mice, there were neither significant main effects nor significant interactions of diet and hormone treatments on SAB and DCX-immunoreactive cell number (Figures 4C,D).

There was no significant effect of age on SAB, although a greater percentage of the late-MA mice $(22.7 \%)$ scored less than $40 \%$ correct alternations, which is uncommon in the early-MA animals (12.5\%), where it occurred almost exclusively in the HFD group. The total number of DCX-positive cells was significantly reduced $\sim 5-10$-fold in the late-MA animals compared to the early-MA mice [Figure 4B vs. Figure 4D, $F_{\text {age }} \times \operatorname{diet}(1,26)=28.5$, $p<0.0001$ ] (see Table 2).

\section{Effects of Diet and Estradiol Treatment on Alzheimer-Related Neuropathology}

The two primary neuropathological hallmarks of $\mathrm{AD}$, deposition of $\mathrm{A} \beta$ and abundant neurons with hyperphosphorylated tau 


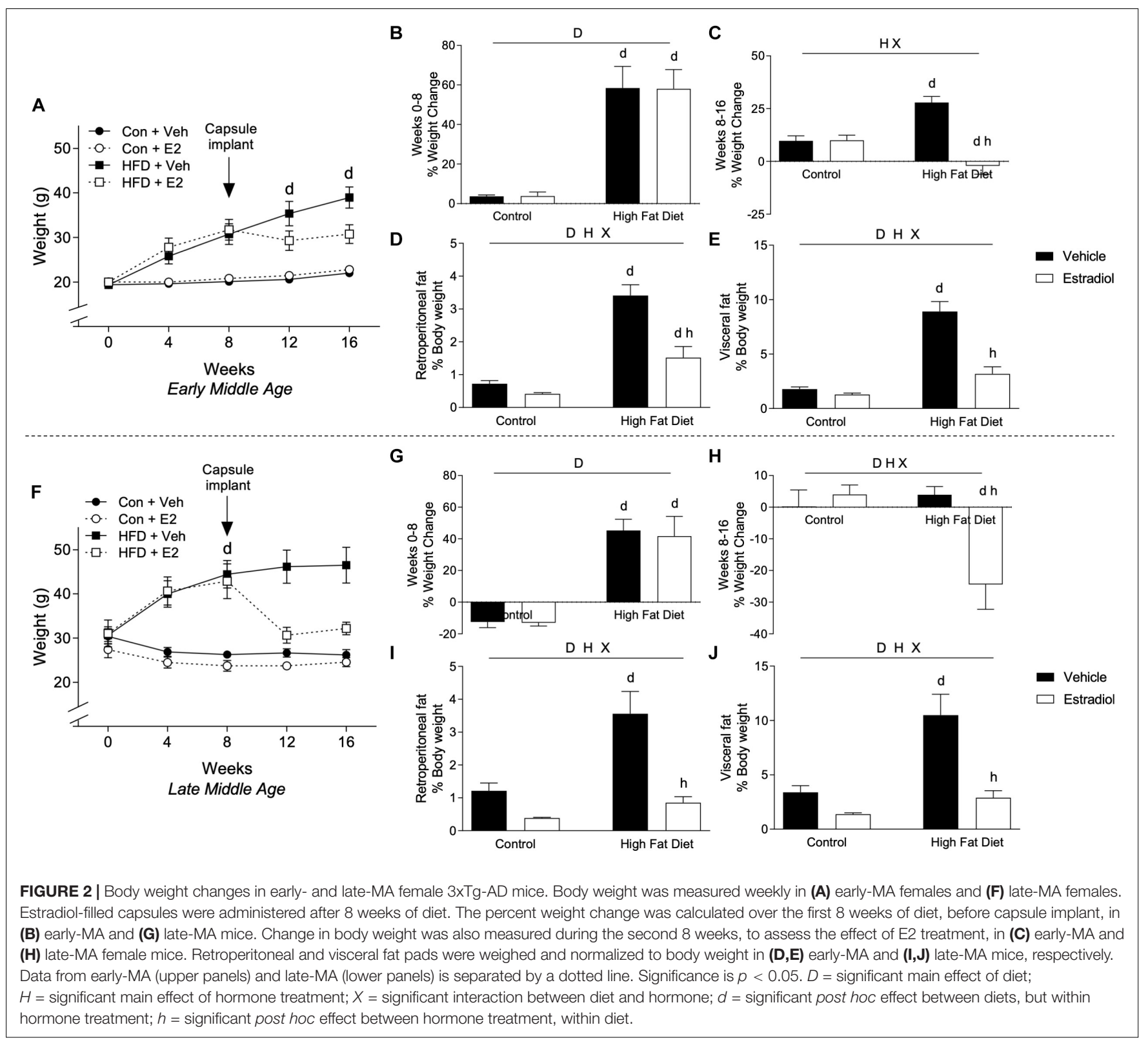

(Iqbal et al., 2009; Crews and Masliah, 2010; Vinters, 2015), are increased with age in 3xTg-AD mice (Oddo et al., 2003). $\mathrm{A} \beta$ deposition was measured by quantifying $\mathrm{A} \beta$ immunoreactive load, and tau pathology was measured by counts of cells immunolabeled with AT8 antibody that specifically recognizes hyperphosphorylated tau species. In the $3 \mathrm{xTg}-\mathrm{AD}$ mouse model of $\mathrm{AD}$, accumulations of tau and $\mathrm{A} \beta$ in the subiculum increased significantly with age, as reported previously (Oddo et al., 2003). Tau increased $\sim 20$-fold in the late-MA animals compared to early-MA females [Figure 5L vs. Figure 5F, $\left.F_{\text {age }} \times \operatorname{diet}(1,26)=57.2, p<0.0001\right] . \mathrm{A} \beta$ load was increased $\sim 5$-fold in the late-MA animals [Figure $5 \mathrm{~K}$ vs. Figure $5 \mathrm{E}$, $\left.F_{\text {age }} \times \operatorname{diet}(1,26)=94.4, p<0.0001\right]$.

In early-MA mice, there was a significant interaction between diet and hormone treatment on $\mathrm{A} \beta$ load [Figures $5 \mathrm{~A}-\mathbf{E}$,
$\left.F_{\text {interaction }}(1,28)=9.9, p<0.01\right]$ in which E2 treatment was associated with reduced $A \beta$ burden in the subiculum specifically in the context of HFD $(p<0.05)$. There were no significant effects of diet or hormone on $A \beta$ load in hippocampus CA1 (data not shown). Numbers of AT8-immunoreactive cells in subiculum showed a statistically non-significant trend of a main effect of hormone treatment $(p=0.07)$ with E2 decreasing tau pathology that was most apparent in the presence of HFD [Figure 5F, $\left.F_{\text {hormone }}(1,27)=3.4, p=0.07\right]$. In the late-MA animals, there were no significant main effects of diet or hormone treatment on $\mathrm{A} \beta$ load in either subiculum (Figures 5G-J,K) or hippocampus CA1 (data not shown). Numbers of AT8-immunoreactive cells showed a significant main effect of diet in which HFD was associated with more hyperphosphorylated tau-positive cells, particularly in the presence of E2 treatment [Figure $5 \mathbf{L}, F_{\text {diet }}(1,23)=6.3, p<0.05$ ]. 
A

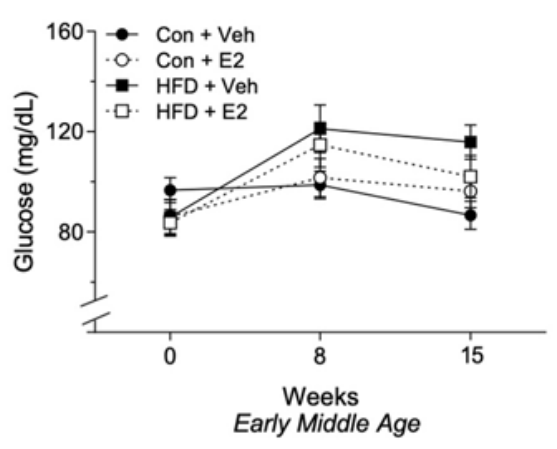

C

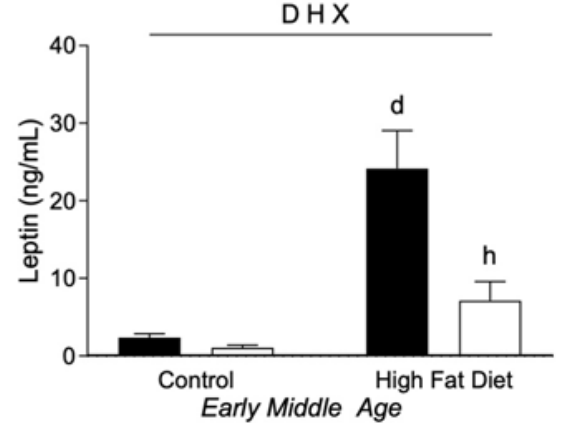

E

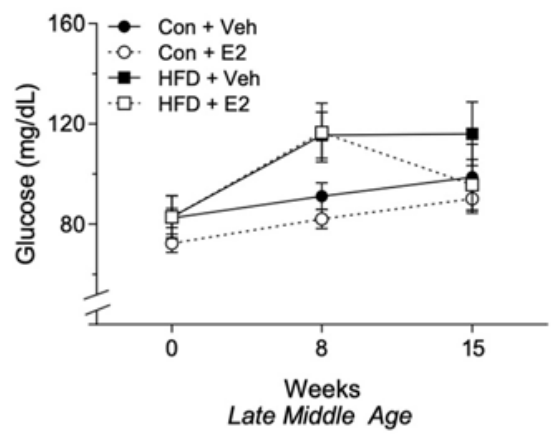

G

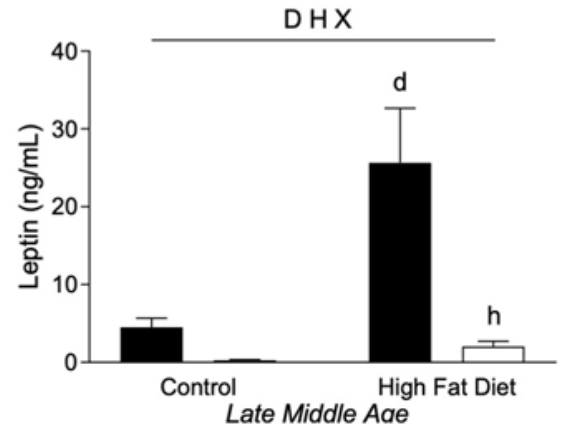

B

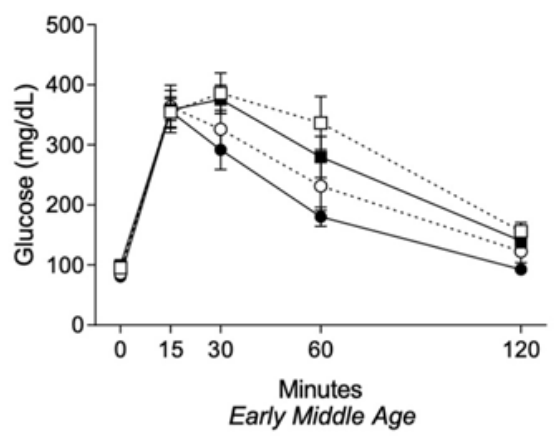

D

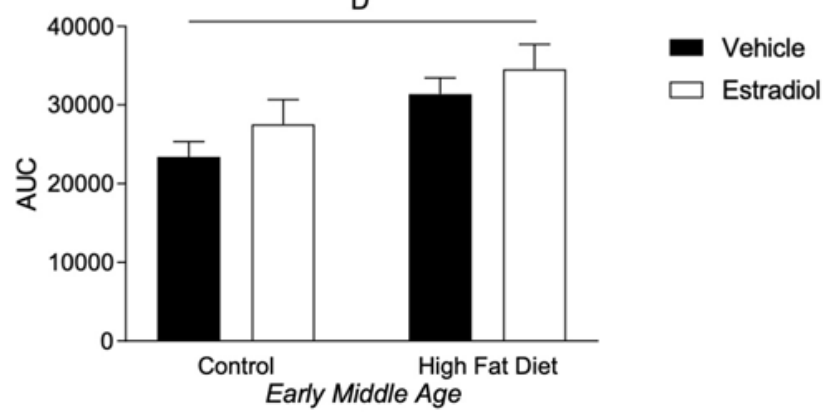

$\mathbf{F}$

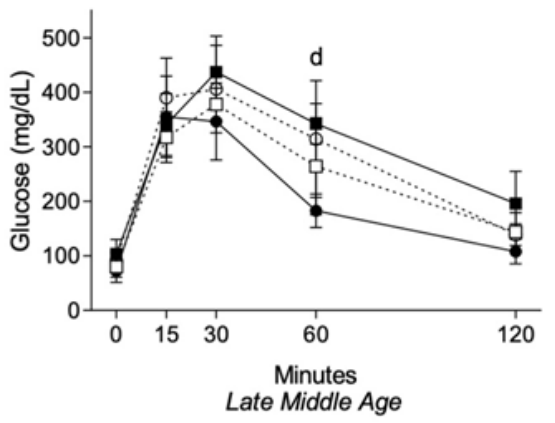

H

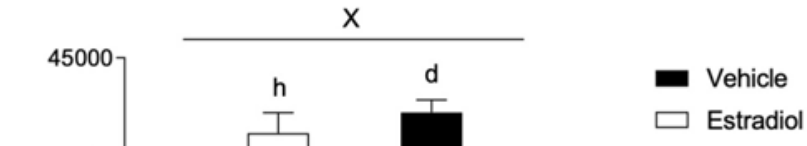

FIGURE 3 | Metabolic effects in early- and late-MA female 3xTg-AD mice. Fasting glucose was measured at weeks 0, 8, and 15. Fasting glucose levels for (A) early-MA and (E) late-MA mice. An oral glucose tolerance test was administered at week 15 in (B) early-MA and (F) late-MA females. Fasting leptin was measured from the plasma collected at week 16 in (C) early-MA and (G) late-MA mice. The area under the curve (AUC) was calculated from the glucose tolerance test in (D) early-MA and $\mathbf{( H )}$ late-MA mice. Significance is $p<0.05 . D=$ significant main effect of diet; $H=$ significant main effect of hormone treatment; $X=$ significant interaction between diet and hormone; $d$ = significant post hoc effect between diets, but within hormone treatment; $h=$ significant post hoc effect between hormone treatment, within diet.

\section{Estradiol Reduces Microglial Activation in Early-MA but Not Late-MA Mice}

Microglial activation is modulated by E2 (Yun et al., 2018; Yilmaz et al., 2019) and is implicated in both regulation of AD-related pathology (Webers et al., 2019) and mediating adverse neural effects of HFD (Thaler et al., 2012; Kim et al., 2019). To investigate potential microglial contributions to observed effects, we quantified microglia cell number and activation phenotype 


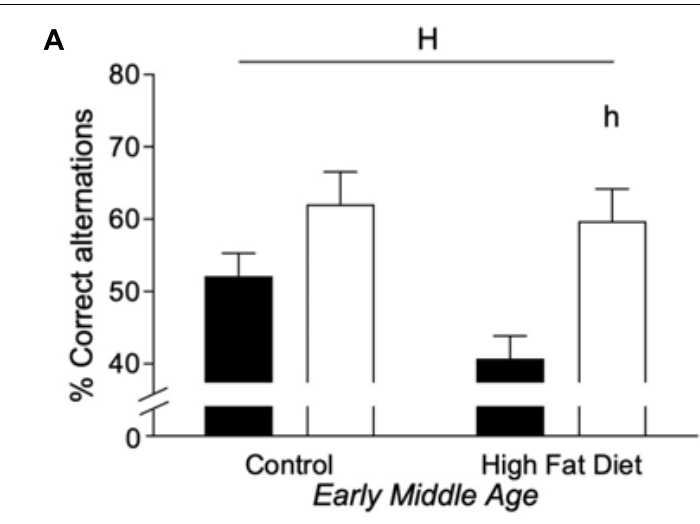

C

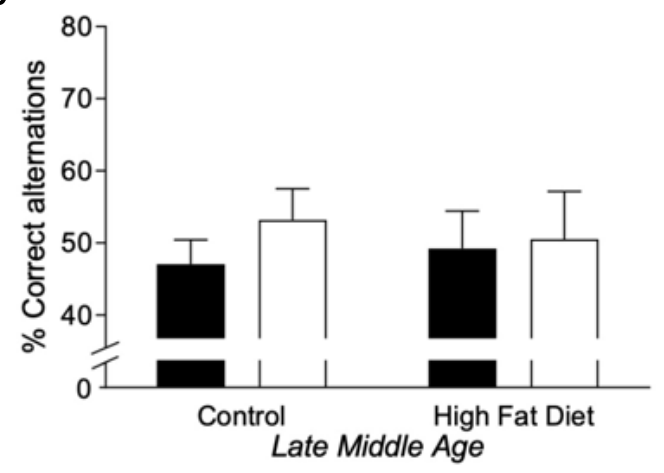

B

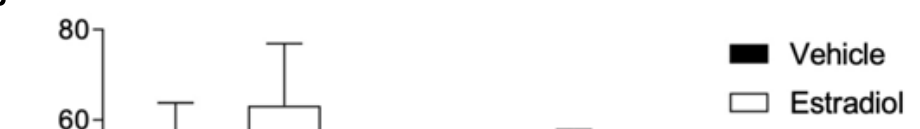

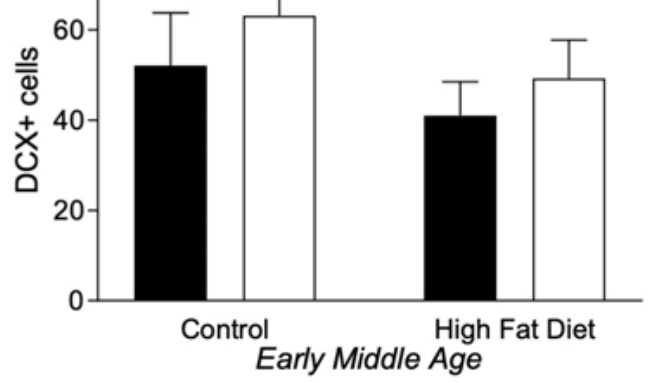

D

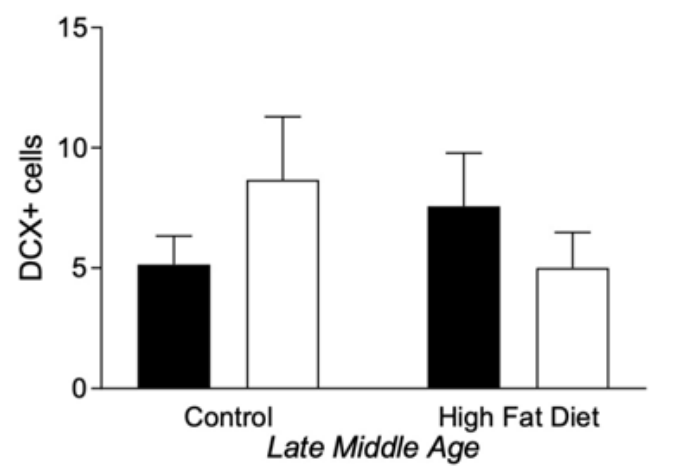

FIGURE 4 | Behavior and neurogenesis in early- and late-MA female 3xTg-AD mice. Correct responses in the spontaneous alternation test were measured in (A) early-MA and (C) late-MA 3xTg-AD females at week 14. Doublecortin-positive cells were counted in the dentate gyrus of the hippocampus at week 16 in (B) early-MA and (D) late-MA mice to measure neurogenesis. Significance is $p<0.05 . H=$ significant main effect of hormone treatment; $h=$ significant post hoc effect between hormone treatment, within diet.

in hippocampus as has been described previously (Christensen and Pike, 2017; Moser and Pike, 2017). In early-MA mice, microglia number showed no significant differences across groups (Figure 6A). However, there were significant main effects of both diet and hormone treatment on microglial activation state such that HFD was associated with increased activation, which was significantly reduced by E2 treatment [Figure 6B, $F_{\text {interaction }}(1,28)=4.2, p<0.05$; HFD + Veh vs. $\mathrm{HFD}+\mathrm{E} 2, p<0.001]$. In late-MA animals, there were significant main effects of diet on both microglia number [Figure 6C, $F_{\text {diet }}(1,22)=4.8, p<0.05$ ] and activation phenotype [Figure 6D, $\left.F_{\text {diet }}(1,22)=6.0, p<0.05\right]$ with HFD-treated mice exhibiting greater microglial density and a higher proportion of activated microglia in the hippocampus than mice maintained on control diet. There were no significant effects of E2 treatment on microglial measures in late-MA mice (Table 1).

There was a significant effect of aging and an age $\mathrm{X}$ diet interaction on microglial number [Figure 6A vs. Figure 6C, $\left.F_{\text {age }} \times \operatorname{diet}(1,25)=28.0, p<0.0001\right]$. Indeed, aging nearly doubled the density of microglia in the hippocampus. The percentage of activated microglia was also significantly increased by aging with an age $\times$ diet and age $\times$ hormone interaction [Figure 6A vs. Figure 6C, $F_{\text {age }} \times$ diet $(1,25)=44.6, p<0.0001$; $F_{\text {age }} \times$ hormone $\left.(1,26)=141.3, p<0.0001\right]$ (see Table 2$)$. The number and activation state of microglia are likely correlated with the increased $A \beta$ load in late-MA animals, but it is unclear if microglia changes are a cause or effect of amyloid changes.

\section{HFD Increases Peripheral Tissue Inflammation in Early-MA and Late-MA Mice}

Diet-induced obesity results in macrophage infiltration and activation in peripheral tissues including adipose tissue that yields an inflammatory state (Huh et al., 2014), an effect that can be attenuated by E2 (Stubbins et al., 2012; Davis et al., 2013). To assess obesity-related macrophage infiltration in visceral adipose tissue, mRNA expression of the macrophage markers CD68 and F4/80 was quantified. In both the early-MA and late-MA groups, HFD significantly increased expression of both macrophage markers [Figure 7A, $F_{\text {diet }}(1,28)=7.1, p<0.05$; Figure $7 \mathbf{B}, F_{\text {diet }}(1,28)=7.5, p<0.05$; Figure $7 D, F_{\text {diet }}(1,23)=9.8$, $p<0.01$; and Figure $\left.7 \mathbf{E}, F_{\text {diet }}(1,23)=4.4, p<0.05\right]$. There were no statistically significant effects of E2 treatment on the HFD-associated increases in CD68 and F4/80 in early-MA and late-MA mice. In addition to macrophage markers, the levels of the pro-inflammatory cytokine IL-6 were assessed by PCR. In early-MA animals, IL-6 expression was significantly increased by diet [Figure 7C, $F_{\text {diet }}(1,28)=4.6, p<0.05$ ] and in late-MA animals IL- 6 was significantly reduced by estradiol 

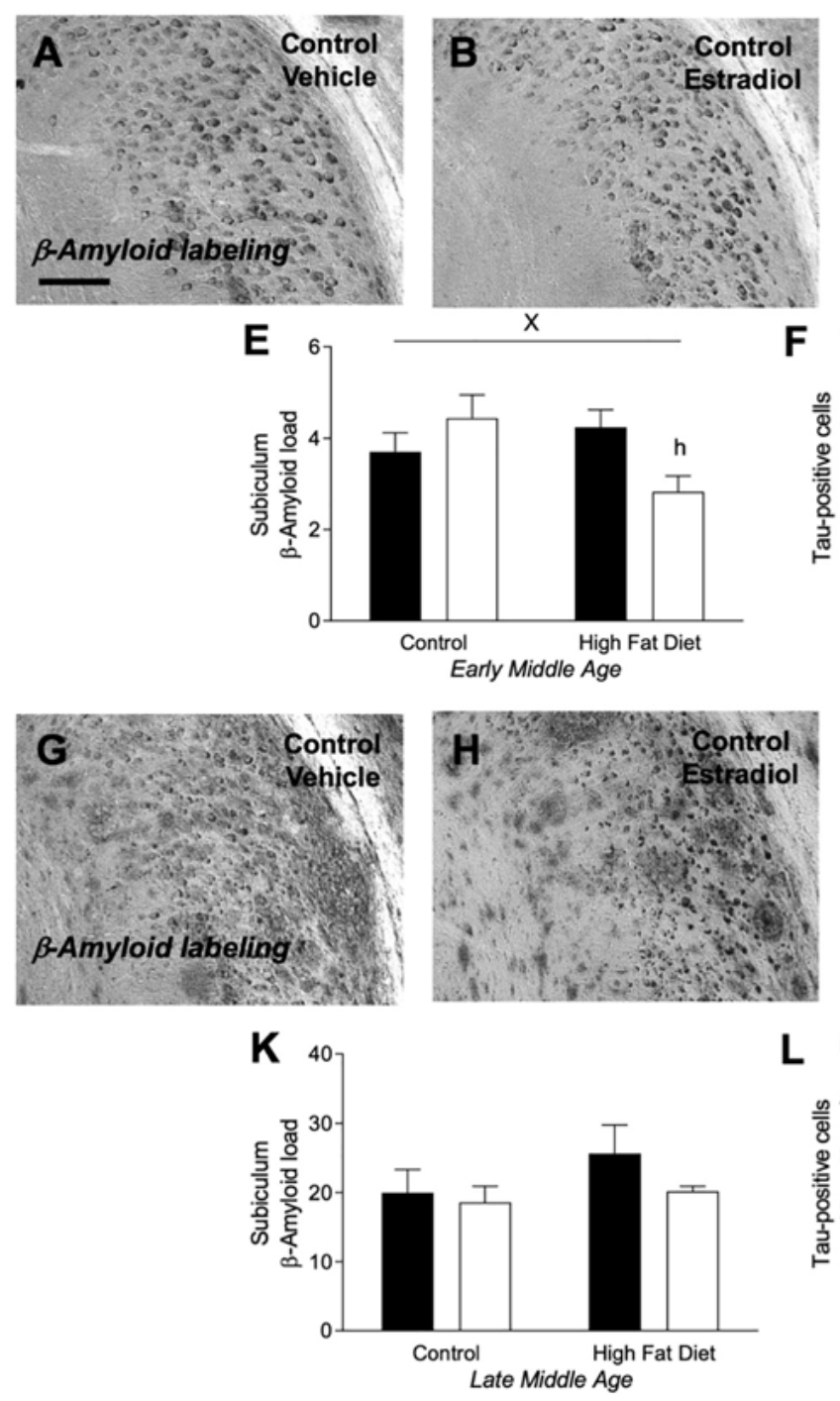

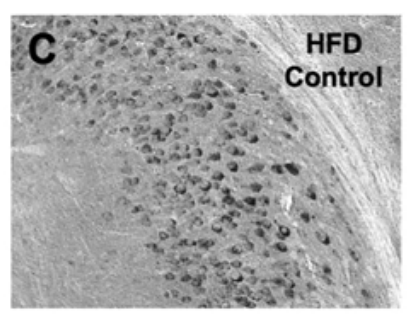

$\mathbf{F}$

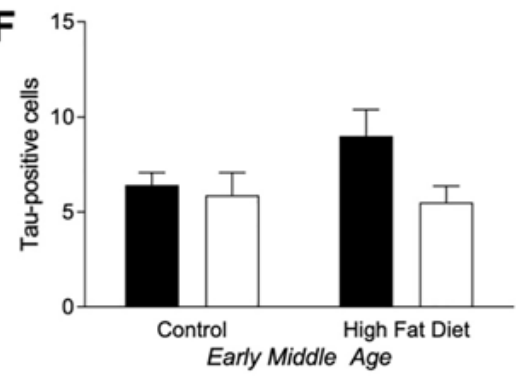

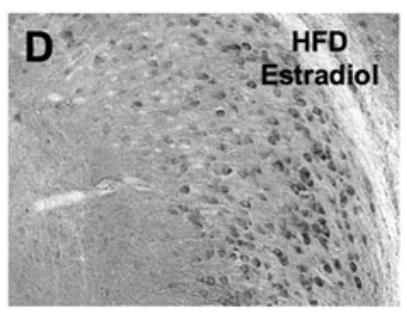

Vehicle

$\square$ Estradiol

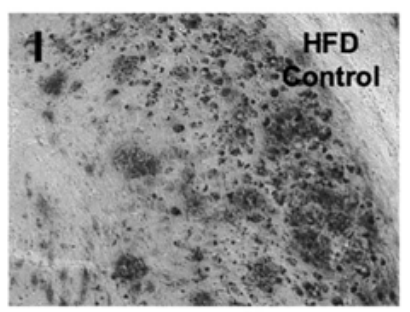

D

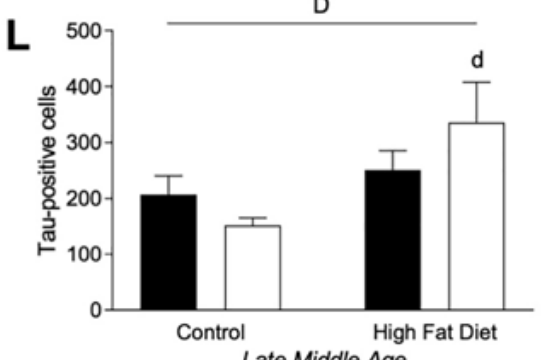

Late Middle Age

FIGURE 5 | Measures of Alzheimer-related neuropathology in early- and late-MA female 3xTg-AD mice. Representative pictures of $\beta$-amyloid immunolabeling in the subiculum of the hippocampal formation from each treatment group in (A-D) early-MA and (G-J) late-MA female mice. $\beta$-Amyloid load was measured in the subiculum of (E) early-MA and (K) late-MA. Tau-immunolabeled cells were counted in the subiculum and CA1 hippocampal regions in (F) early-MA and (L) late-MA mice. Significance is $p<0.05 . D=$ significant main effect of diet; $H=$ significant main effect of hormone treatment; $X=$ significant interaction between diet and hormone; $d$ = significant post hoc effect between diets, but within hormone treatment; $h=$ significant post hoc effect between hormone treatment, within diet.

treatment but was not significantly affected by diet [Figure $7 \mathbf{F}$, $\left.F_{\text {hormone }}(1,23)=5.2, p<0.05\right]$.

There was no significant, independent effect of aging on macrophage markers in visceral adipose tissue. However, when all the animals were compared together, a significant effect of diet and a significant interaction between diet and hormones was seen that was not apparent when the ages were compared separately [Figure 7A vs. Figure 7D, $F_{\text {diet }}(1,51)=17.3, p<0.001$; $F_{\text {diet }} \times$ hormone $(1,51)=5.4, p<0.05$; Figure $7 \mathbf{B}$ vs. Figure $7 \mathbf{E}$, $F_{\text {diet }}(1,51)=11.1, p<0.01 ; F_{\text {diet }} \times$ hormone $\left.(1,51)=4.4, p<0.05\right]$. When comparing adipose levels of IL-6 across aging, there is a trend for HFD to increase IL-6 levels and a statistically nonsignificant trend toward an age $\mathrm{X}$ diet interaction in which early-MA animals show a greater response to diet than late-MA animals [Figure $7 \mathrm{C}$ vs. Figure $7 \mathbf{F}, F_{\text {diet }}(1,51)=3.1, p=0.09$; $\left.F_{\text {age }} \times \operatorname{diet}(1,51)=3.3, p=0.08\right]$.

\section{DISCUSSION}

Obesity is a significant and highly prevalent risk factor for poor health outcomes and increased vulnerability to several diseases, including AD (Moser and Pike, 2016; Pugazhenthi et al., 2017; Alford et al., 2018). Like other AD risk factors, the deleterious effects associated with obesity can be positively and negatively modulated by several factors, underscoring the need to understand how obesity interacts with other regulators of $\mathrm{AD}$. In this study, we looked at how obesity, aging, and 

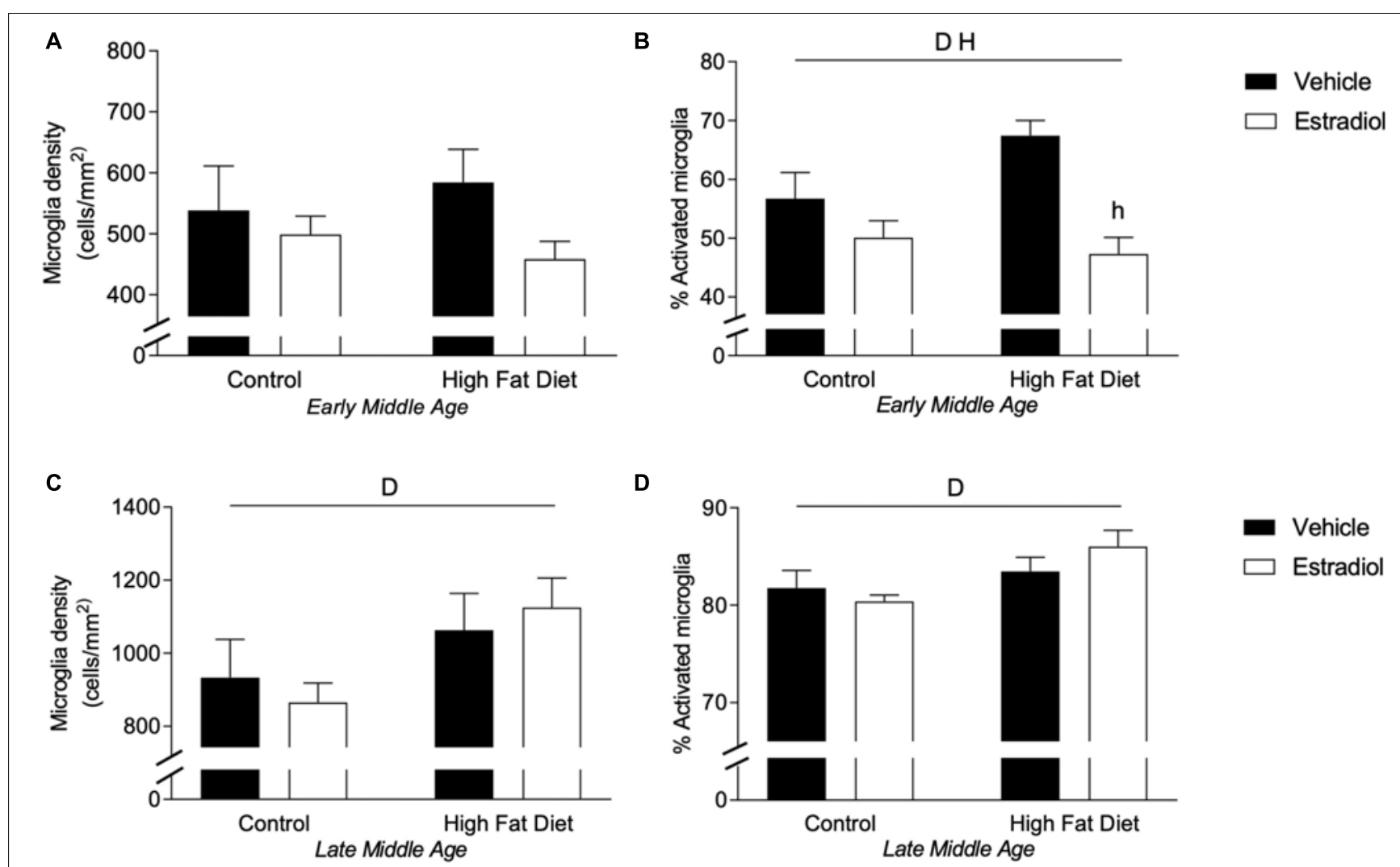

FIGURE 6 | Microglial density and activation in the hippocampus of female 3xTg-AD mice. Microglia in the hippocampus were immunostained with Iba-1 antibody, counted, and morphologically characterized as either activated or resting. Microglia cell density was measured in (A) early-MA and (C) late-MA mice. The percent of microglia exhibiting activated morphology was determined in (B) early-MA and (D) late-MA mice. Significance is $p<0.05$. $D=$ significant main effect of diet; $H=$ significant main effect of hormone treatment.

estradiol interact to affect AD neuropathology. In particular, this study assessed the protective effects of E2 against metabolic and AD-related outcomes associated with obesity during the initial versus late stages of middle age in female $3 \mathrm{xTg}$-AD mice. Prior related studies largely delivered E2 simultaneously with HFD (Litwak et al., 2014; Yasrebi et al., 2017), and thereby assessed the preventive effects of E2 against obesity. Here, E2 was initiated after the establishment of diet-induced obesity, which considers potential treatment effects of E2 in the context of an existing $\mathrm{AD}$ risk factor. In early-MA, we observed that E2 effectively reduced systemic effects of obesity, improved working memory performance, lessened $\mathrm{A} \beta$ burden in subiculum, and decreased an activated microglial phenotype. In late-MA, metabolic benefits of E2 were also found, however there was an absence of protection against $\mathrm{AD}$-related neuropathology and cognitive impairment.

Metabolic benefits of estrogens may be retained during aging. The depletion of estrogens during menopause is associated with numerous changes throughout the body, including increased adiposity and associated metabolic impairments (Wend et al., 2012). Reduced estradiol levels during the menopause transition have been linked to increased risk of type 2 diabetes (Park et al., 2017). Postmenopausal women treated with HT exhibit reduced adiposity (Papadakis et al., 2018) and metabolic benefits including decreases in fasting glucose and plasma lipids and reduced type 2 diabetes risk (Andersson et al., 1997; Kanaya et al., 2003; Margolis et al., 2004; Mauvais-Jarvis et al., 2017). Our data show that late-MA mice had significantly higher mass than early-MA animals at the start of the experiment, suggesting that the combination of reproductive and chronological aging in female mice models aspects of the increased adiposity observed in postmenopausal women. More importantly, E2 administered after the development of obesity was able to mitigate weight gain both in early-MA and late-MA animals, suggesting retained functionality of peripheral estrogen signaling across middle age. Interestingly, late-MA animals showed a greater response to E2 than early-MA mice in terms of body mass. In addition, plasma leptin concentration was significantly increased by diet and reduced by E2 in both early-MA and late-MA mice. These data complement prior findings that E2 treatment initiated simultaneously with obesogenic diet attenuated adiposity in advanced middle-aged female mice and that E2 late in life can reduce metabolic dysfunction induced by HFD (Bryzgalova et al., 2008; Christensen and Pike, 2017). However, recent studies in aged mice (Farhadi et al., 2020) and macaques (Purnell et al., 2019) maintained on an obesogenic diet indicate loss of E2 efficacy in reducing body mass and improving metabolic measures. Unclear from both the human and animal literatures is the relationship between chronological and reproductive aging 


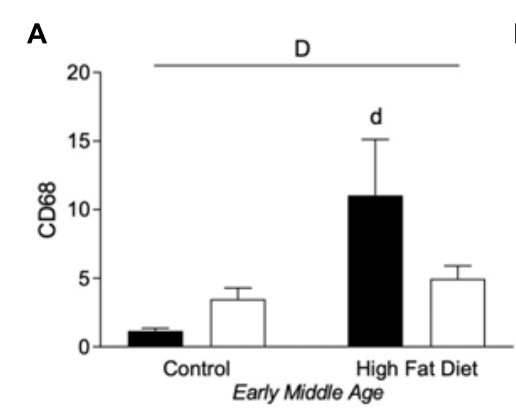

D

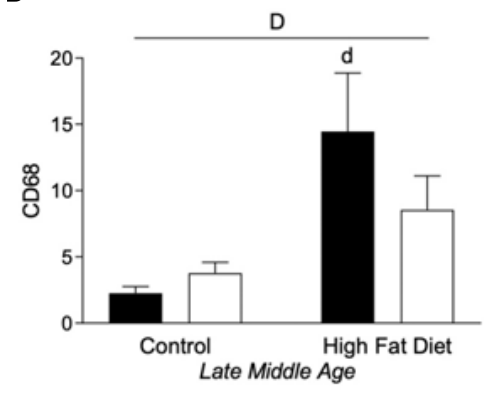

B

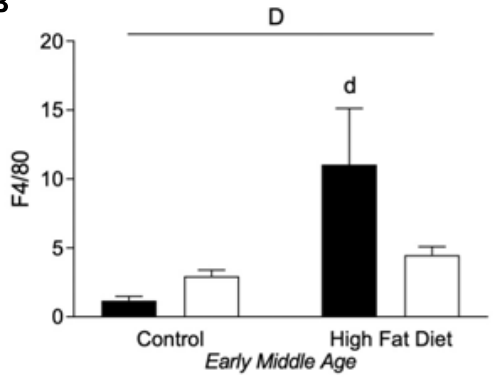

E

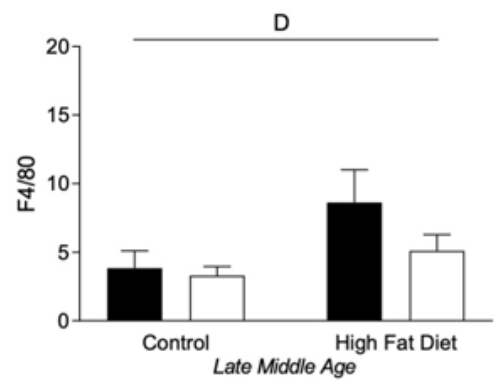

C

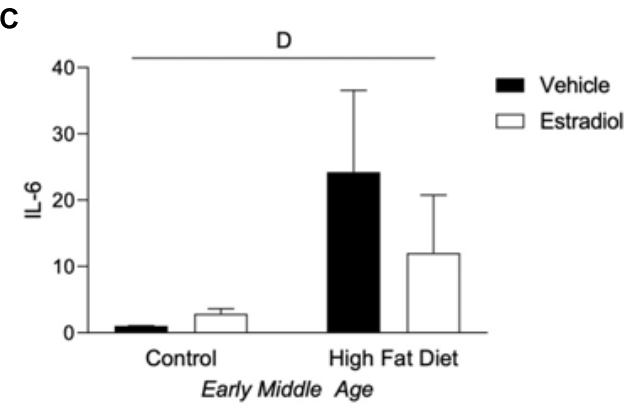

$\mathbf{F}$

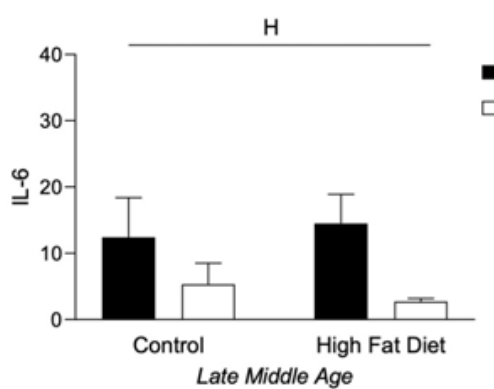

FIGURE 7 | Macrophage markers in adipose tissue in early- and late-MA female 3xTg-AD mice. Relative mRNA expression of the macrophage markers CD68 and F4/80 was measured in visceral adipose tissue from (A,B) early-MA and (D,E) late-MA mice, respectively. The cytokine IL-6 mRNA expression was measured from adipose tissue in (C) early-MA and (F) late-MA mice. Significance is $p<0.05 . D=$ significant main effect of diet; $H=$ significant main effect of hormone treatment; $d=$ significant post hoc effect between diets, but within hormone treatment.

in regulating E2 efficacy and how this may vary across tissues. Prior work has demonstrated the importance of reproductive over chronological aging for some neural measures in early middle-aged female rats (Yin et al., 2015) and women (Mosconi et al., 2017). Our current observations that late-MA mice retain beneficial E2 effects for peripheral metabolic outcomes but not neural endpoints suggest key differences across tissues. These are critical issues that require additional study.

The neuroprotective actions of estrogens appear to be adversely affected by chronological and reproductive aging. In women, there is an extensive but conflicting literature concerning the ability of HT to reduce risks of AD and related dementias. Although the controversy has yet to be resolved definitively, a leading perspective is that neuroprotective estrogen actions are diminished across the menopause transition such that there may be a several year window of opportunity, beginning at perimenopause, during which HT exhibits transient neural efficacy (Maki, 2006; Henderson et al., 2016). After this period, HT is thought to exert neutral or perhaps harmful neural effects (Henderson et al., 2005; Whitmer et al., 2011; Shao et al., 2012). Experimental animal studies parallel aspects of the human literature in that E2 treatment yields significant neural benefits in middle-aged females across several rodent paradigms (Pike et al., 2009), outcomes that are often attenuated in aged animals (Aenlle and Foster, 2010; Liu et al., 2015). Note, however, that E2 can exert positive neural effects, including improved behavioral performance, even in aged female mice (Frick et al., 2002; Vaucher et al., 2002). Unclear is whether and how obesity affects the trend of age-related decreases in neural efficacy of E2. In the current study, E2 treatment in early-MA mice under Con diet yielded only statistically non-significant trends toward improved behavioral performance and reduced $\mathrm{A} \beta$. These observations are in contrast to our previous findings of E2 in gonadally intact (Christensen and Pike, 2017) and ovariectomized (Carroll et al., 2007) female $3 x$ Tg-AD mice, perhaps owing to the comparatively short treatment period in the present study. In the context of HFD, early-MA mice treated with E2 showed improved behavior, reduced $\mathrm{A} \beta$ burden in subiculum, and a non-significant trend toward lower phospho-tau labeling. Importantly, lateMA mice treated with E2 showed a quite different pattern with no improvements in behavior and $A \beta$ and a significant worsening of phospho-tau. Together, these findings suggest that neuroprotective effects of E2 are retained in early-MA even in the presence of a preexisting obese state. In apparent contrast to our observations, recent work in aged female macaques showed that positive effects of E2 on object recognition memory were blunted by obesogenic diet (Bethea et al., 2017). This lack of concordance suggests the possibility of a complex relationship between obesity and E2 neural efficacy during aging that may be affected by several parameters, including specific aspects of diet, aging, and neural measure.

Coincident impairments in metabolism and brain functions suggest possible mechanistic relationships. Indeed, metabolic dysfunction has been widely hypothesized to be a key mechanism by which obesity induces neural deficits and increases AD risk (O'Brien et al., 2017; Pugazhenthi et al., 2017; Alford et al., 2018). Consistent with this position, previous work has shown interventions that reduce obesity in transgenic mouse models 
of $\mathrm{AD}$ also improve behavioral and pathological outcomes (Maesako et al., 2012; Walker et al., 2017; Yeh et al., 2017; Ettcheto et al., 2018). However, the current findings are not supportive of direct associations between improved metabolic profile and reduced $\mathrm{AD}$-related indices. E2 significantly reduced body mass, adiposity, and circulating leptin levels in both early-MA and lateMA yet was associated with improved behavioral performance and lower $A \beta$ burden only in early-MA. The observation that metabolic but not neural benefits of E2 persisted in late-MA argues against metabolic regulation as a primary component underlying E2 protection against AD-related outcomes.

Aside from improving metabolic indices, estrogens may also combat neural consequences of obesity by regulating systemic and CNS inflammatory tone. Diet-induced obesity results in activated microglial phenotypes, which in turn mediate, at least in part, damaging, pro-inflammatory effects of obesity on the brain (Hao et al., 2016; André et al., 2017). Importantly, impaired microglial functions are increasingly implicated as key regulators of AD pathogenesis (Kaur et al., 2019; Webers et al., 2019). Estradiol represents a compelling approach in combating effects of obesity because it can attenuate disease-promoting microglial actions both directly by regulating microglia (Villa et al., 2016; Butler et al., 2020) and indirectly by reducing adiposity (Brown et al., 2010). We observed an obesity-induced increase in an activated microglial phenotype at both early-MA and late-MA. Importantly, E2 reversed this increase in microglial activation in early-MA but had no significant effect in late-MA. These data suggest that neural E2 regulation of microglia in female mice is sensitive to reproductive and/or chronological aging with efficacy largely absent by late middle-age. Interestingly, this pattern corresponds with the observed age-dependence of E2 protection against behavioral impairment and $A \beta$ burden, suggesting perhaps that reduction of microglial activation may contribute to the mechanism(s) by which $\mathrm{E} 2$ protects against $\mathrm{AD}$-related outcomes in obese female $3 \mathrm{xTg}$-AD mice. Although estrogens are known to regulate peripheral inflammatory tone (Kovats, 2015; Klein and Flanagan, 2016), our limited assessment showed that E2 treatment of HFD mice yielded only a nonsignificant trend toward blunting the obesity-induced increase in macrophage markers in adipose in early-MA, which was less apparent in late-MA. These observations are not supportive of a possible mechanistic role of reduced peripheral inflammatory tone mediating neural effects of E2, though the restricted assessment of systemic inflammation status precludes a definitive conclusion.

In summary, these results add to an important and growing literature defining the impact of aging on the ability of estrogenbased therapies to protect against $\mathrm{AD}$. The key finding is that, in the context of preexisting obesity, E2 treatment improves cognition and reduces $A \beta$ burden in mice at chronological ages associated with early but not late reproductive senescence. This

\section{REFERENCES}

Aenlle, K. K., and Foster, T. C. (2010). Aging alters the expression of genes for neuroprotection and synaptic function following acute estradiol treatment. Hippocampus 20, 1047-1060. doi: 10.1002/hipo.20703 finding supports the "window of opportunity" position that HT in aging women is most likely to yield protective brain effects when initiated near perimenopause but absent or even deleterious outcomes when delivered several years after the menopause transition (Whitmer et al., 2011; Shao et al., 2012; Henderson et al., 2016). Further, because increasing adiposity is associated with chronological and reproductive aging in women, the findings provide preclinical evidence that HT may provide effective intervention for women that are overweight or obese, which are established $\mathrm{AD}$ risk factors. The results also provide clues to possible mechanisms contributing to E2 protection against obesity. To the extent that deleterious effects of obesity drive neural dysfunction as a result of systemic metabolic impairment, the current findings suggest that E2 may benefit brain health and reduce $\mathrm{AD}$ risk well into middle age as a consequence of its ability to lower adiposity and improve metabolic function in the context of obesity. However, the discordance between E2 protection against metabolic versus neural indices in late reproductive senescence argue that other actions, perhaps including regulation of microglial activation states, play more significant roles in protections against $\mathrm{AD}$. Continued investigation of the interactions among E2, aging, obesity, and other $\mathrm{AD}$ risk factors promises to improve the selective use of HT to reduce dementia risk.

\section{DATA AVAILABILITY STATEMENT}

The datasets generated for this study are available on request to the corresponding author.

\section{ETHICS STATEMENT}

The animal study was reviewed and approved by the Institutional Animal Care and Use Committee of the University of Southern California. Procedures were performed under the supervision of university veterinarians.

\section{AUTHOR CONTRIBUTIONS}

AC and CP designed the study, analyzed the results, and wrote the manuscript. AC performed the experiments. JL conducted and analyzed some of the PCR experiments.

\section{FUNDING}

This work was supported by NIH grant AG026572 (RD Brinton and CJP/Project 3). AC was supported in part by NIH National Institute on Aging grant T32 AG052374.

Alford, S., Patel, D., Perakakis, N., and Mantzoros, C. S. (2018). Obesity as a risk factor for Alzheimer's disease: weighing the evidence. Obes. Rev. 19, 269-280. doi: 10.1111/obr.12629

Alzheimer's Association (2018). 2018 Alzheimer's disease facts and figures. Alzheimers Dement. 14, 367-429. doi: 10.1016/j.jalz.2018.02.001 
Andersson, B., Mattsson, L. A., Hahn, L., Mårin, P., Lapidus, L., Holm, G., et al. (1997). Estrogen replacement therapy decreases hyperandrogenicity and improves glucose homeostasis and plasma lipids in postmenopausal women with noninsulin-dependent diabetes mellitus. J. Clin. Endocrinol. Metab. 82, 638-643. doi: 10.1210/jcem.82.2.3746

André, C., Guzman-Quevedo, O., Rey, C., Rémus-Borel, J., Clark, S., CastellanosJankiewicz, A., et al. (2017). Inhibiting microglia expansion prevents dietinduced hypothalamic and peripheral inflammation. Diabetes 66, 908-919. doi: 10.2337/db16-0586

Bangen, K. J., Armstrong, N. M., Au, R., and Gross, A. L. (2019). Metabolic syndrome and cognitive trajectories in the framingham offspring study. J. Alzheimers Dis. 71, 931-943. doi: 10.3233/JAD-190261

Barker, J. M., and Galea, L. A. M. (2008). Repeated estradiol administration alters different aspects of neurogenesis and cell death in the hippocampus of female, but not male, rats. Neuroscience 152, 888-902. doi: 10.1016/j.neuroscience.2007. 10.071

Bethea, C. L., Mueller, K., Reddy, A. P., Kohama, S. G., and Urbanski, H. F. (2017). Effects of obesogenic diet and estradiol on dorsal raphe gene expression in old female macaques. PLoS ONE 12:e178788. doi: 10.1371/journal.pone.0178788

Bove, R., Secor, E., Chibnik, L. B., Barnes, L. L., Schneider, J. A., Bennett, D. A., et al. (2014). Age at surgical menopause influences cognitive decline and Alzheimer pathology in older women. Neurology 82, 222-229. doi: 10.1212/ WNL.0000000000000033

Brown, L. M., Gent, L., Davis, K., and Clegg, D. J. (2010). Metabolic impact of sex hormones on obesity. Brain Res. 1350, 77-85. doi: 10.1016/j.brainres.2010. 04.056

Bryzgalova, G., Lundholm, L., Portwood, N., Gustafsson, J.-A., Khan, A., Efendic, S., et al. (2008). Mechanisms of antidiabetogenic and body weight-lowering effects of estrogen in high-fat diet-fed mice. Am. J. Physiol. Endocrinol. Metab. 295, E904-E912. doi: 10.1152/ajpendo.90248.2008

Butler, M. J., Perrini, A. A., and Eckel, L. A. (2020). Estradiol treatment attenuates high fat diet-induced microgliosis in ovariectomized rats. Horm. Behav. 120:104675. doi: 10.1016/j.yhbeh.2020.104675

Carroll, J. C., Rosario, E. R., Chang, L., Stanczyk, F. Z., Oddo, S., LaFerla, F. M., et al. (2007). Progesterone and estrogen regulate Alzheimer-like neuropathology in female 3xTg-AD mice. J. Neurosci. 27, 13357-13365. doi: 10.1523/JNEUROSCI. 2718-07.2007

Christensen, A., and Pike, C. J. (2015). Menopause, obesity and inflammation: interactive risk factors for Alzheimer's disease. Front. Aging Neurosci. 7:130. doi: 10.3389/fnagi.2015.00130

Christensen, A., and Pike, C. J. (2017). Age-dependent regulation of obesity and Alzheimer-related outcomes by hormone therapy in female 3xTg-AD mice. PLoS ONE 12:e0178490. doi: 10.1371/journal.pone.0178490

Christensen, A., and Pike, C. J. (2018). APOE genotype affects metabolic and Alzheimer-related outcomes induced by Western diet in female EFAD mice. FASEB J. 33, 4054-4066. doi: 10.1096/fj.201801756R

Clegg, D., Hevener, A. L., Moreau, K. L., Morselli, E., Criollo, A., Van Pelt, R. E., et al. (2017). Sex hormones and cardiometabolic health: role of estrogen and estrogen receptors. Endocrinology 158, 1095-1105. doi: 10.1210/en.2016-1677

Crews, L., and Masliah, E. (2010). Molecular mechanisms of neurodegeneration in Alzheimer's disease. Hum. Mol. Genet. 19, R12-R20. doi: 10.1093/hmg/ddq160

Davis, K. E., Neinast, D. M., Sun, K., Skiles, W. M., Bills, J., Zehr, J. A., et al. (2013). The sexually dimorphic role of adipose and adipocyte estrogen receptors in modulating adipose tissue expansion, inflammation, and fibrosis. Mol. Metab. 2, 227-242. doi: 10.1016/j.molmet.2013.05.006

Depypere, H., Vierin, A., Weyers, S., and Sieben, A. (2016). Alzheimer's disease, apolipoprotein E and hormone replacement therapy. Maturitas 94, 98-105. doi: 10.1016/j.maturitas.2016.09.009

Ettcheto, M., Sánchez-López, E., Gómez-Mínguez, Y., Cabrera, H., Busquets, O., Beas-Zarate, C., et al. (2018). Peripheral and central effects of memantine in a mixed preclinical mice model of obesity and familial alzheimer's disease. $\mathrm{Mol}$. Neurobiol. 55, 7327-7339. doi: 10.1007/s12035-018-0868-4

Farhadi, Z., Khaksari, M., Azizian, H., Dabiri, S., Fallah, H., and Nozari, M. (2020). Aging is associated with loss of beneficial effects of estrogen on leptin responsiveness in mice fed high fat diet: role of estrogen receptor $\alpha$ and cytokines. Mech. Ageing Dev. 186, 111198. doi: 10.1016/j.mad.2019.111198

Felicio, L. S., Nelson, J. F., and Finch, C. E. (1984). Longitudinal studies of estrous cyclicity in aging C57BL/6J mice: II. Cessation of cyclicity and the duration of persistent vaginal cornification. Biol. Reprod. 31, 446-453. doi: 10.1095/ biolreprod31.3.446

Finch, C. E. (2014). The menopause and aging, a comparative perspective. J. Steroid Biochem. Mol. Biol. 142, 132-141. doi: 10.1016/j.jsbmb.2013.03.010

Frick, K. M., Fernandez, S. M., and Bulinski, S. C. (2002). Estrogen replacement improves spatial reference memory and increases hippocampal synaptophysin in aged female mice. Neuroscience 115, 547-558. doi: 10.1016/s0306-4522(02) 00377-9

Frick, K. M., Kim, J., Tuscher, J. J., and Fortress, A. M. (2015). Sex steroid hormones matter for learning and memory: estrogenic regulation of hippocampal function in male and female rodents. Learn. Mem. 22, 472-493. doi: 10.1101/lm.0372 67.114

Hamoda, H., Panay, N., Arya, R., Savvas, M., and on behalf of The British Menopause Society and Women's Health Concern (2016). The British Menopause Society \& Women's Health Concern 2016 recommendations on hormone replacement therapy in menopausal women. Post Reprod. Health 22, 165-183. doi: 10.1177/2053369116680501

Hao, S., Dey, A., Yu, X., and Stranahan, A. M. (2016). Dietary obesity reversibly induces synaptic stripping by microglia and impairs hippocampal plasticity. Brain Behav. Immun. 51, 230-239. doi: 10.1016/j.bbi.2015.08.023

Henderson, V. W., Benke, K. S., Green, R. C., Cupples, L. A., Farrer, L. A., Mirage Study, et al. (2005). Postmenopausal hormone therapy and Alzheimer's disease risk: interaction with age. J. Neurol. Neurosurg. Psychiatry 76, 103-105. doi: 10.1136/jnnp.2003.024927

Henderson, V. W., St John, J. A., Hodis, H. N., McCleary, C. A., Stanczyk, F. Z., Shoupe, D., et al. (2016). Cognitive effects of estradiol after menopause: a randomized trial of the timing hypothesis. Neurology 87, 699-708. doi: 10.1212/ WNL.0000000000002980

Hersi, M., Irvine, B., Gupta, P., Gomes, J., Birkett, N., and Krewski, D. (2017). Risk factors associated with the onset and progression of Alzheimer's disease: a systematic review of the evidence. Neurotoxicology 61, 143-187. doi: 10.1016/ j.neuro.2017.03.006

Hughes, R. N. (2004). The value of spontaneous alternation behavior (SAB) as a test of retention in pharmacological investigations of memory. Neurosci. Biobehav. Rev. 28, 497-505. doi: 10.1016/j.neubiorev.2004.06.006

Huh, J. Y., Park, Y. J., Ham, M., and Kim, J. B. (2014). Crosstalk between adipocytes and immune cells in adipose tissue inflammation and metabolic dysregulation in obesity. Mol. Cells 37, 365-371. doi: 10.14348/molcells.2014.0074

Iqbal, K., Liu, F., Gong, C.-X., Alonso, A. D. C., and Grundke-Iqbal, I. (2009). Mechanisms of tau-induced neurodegeneration. Acta Neuropathol. 118, 53-69. doi: 10.1007/s00401-009-0486-3

Jang, H., Bae, J. B., Dardiotis, E., Scarmeas, N., Sachdev, P. S., Lipnicki, D. M., et al. (2018). Differential effects of completed and incomplete pregnancies on the risk of Alzheimer disease. Neurology 91, e643-e651. doi: 10.1212/WNL. 000000000000600

Kanaya, A. M., Herrington, D., Vittinghoff, E., Lin, F., Grady, D., Bittner, V., et al. (2003). Glycemic effects of postmenopausal hormone therapy: the Heart and Estrogen/progestin Replacement Study. A randomized, double-blind, placebocontrolled trial. Ann. Intern. Med. 138, 1-9.

Kaur, D., Sharma, V., and Deshmukh, R. (2019). Activation of microglia and astrocytes: a roadway to neuroinflammation and Alzheimer's disease. Inflammopharmacology 27, 663-677. doi: 10.1007/s10787-019-00580-x

Kim, J. D., Yoon, N. A., Jin, S., and Diano, S. (2019). Microglial UCP2 mediates inflammation and obesity induced by high-fat feeding. Cell Metab. 30, 952962.e5. doi: 10.1016/j.cmet.2019.08.010

Klein, S. L., and Flanagan, K. L. (2016). Sex differences in immune responses. Nat. Rev. Immunol. 16, 626-638. doi: 10.1038/nri.2016.90

Kovats, S. (2015). Estrogen receptors regulate innate immune cells and signaling pathways. Cell Immunol. 294, 63-69. doi: 10.1016/j.cellimm.2015.01.018

Lalonde, R. (2002). The neurobiological basis of spontaneous alternation. Neurosci. Biobehav. Rev. 26, 91-104. doi: 10.1016/s0149-7634(01)00041-0

Li, R., and Singh, M. (2014). Sex differences in cognitive impairment and Alzheimer's disease. Front. Neuroendocrinol. 35:385-403. doi: 10.1016/j.yfrne. 2014.01.002

Litwak, S. A., Wilson, J. L., Chen, W., Garcia-Rudaz, C., Khaksari, M., Cowley, M. A., et al. (2014). Estradiol prevents fat accumulation and overcomes leptin resistance in female high-fat diet mice. Endocrinology 155, 4447-4460. doi: $10.1210 /$ en.2014-1342 
Liu, J., Lin, H., Huang, Y., Liu, Y., Wang, B., and Su, F. (2015). Cognitive effects of long-term dydrogesterone treatment used alone or with estrogen on rat menopausal models of different ages. Neuroscience 290, 103-114. doi: 10.1016/ j.neuroscience.2015.01.042

Lobo, R. A. (2017). Hormone-replacement therapy: current thinking. Nat. Rev. Endocrinol. 13, 220-231. doi: 10.1038/nrendo.2016.164

Lobo, R. A., Pickar, J. H., Stevenson, J. C., Mack, W. J., and Hodis, H. N. (2016). Back to the future: hormone replacement therapy as part of a prevention strategy for women at the onset of menopause. Atherosclerosis 254, 282-290. doi: 10.1016/j.atherosclerosis.2016.10.005

Maesako, M., Uemura, K., Kubota, M., Kuzuya, A., Sasaki, K., Hayashida, N., et al. (2012). Exercise is more effective than diet control in preventing high fat diet-induced $\beta$-amyloid deposition and memory deficit in amyloid precursor protein transgenic mice. J. Biol. Chem. 287, 23024-23033. doi: 10.1074/jbc. M112.367011

Maki, P. M. (2006). Hormone therapy and cognitive function: is there a critical period for benefit? Neuroscience 138, 1027-1030. doi: 10.1016/j.neuroscience. 2006.01.001

Margolis, K. L., Bonds, D. E., Rodabough, R. J., Tinker, L., Phillips, L. S., Allen, C., et al. (2004). Effect of oestrogen plus progestin on the incidence of diabetes in postmenopausal women: results from the Women's Health Initiative Hormone Trial. Diabetologia 47, 1175-1187. doi: 10.1007/s00125-004-1448-x

Marjoribanks, J., Farquhar, C. M., Roberts, H., and Lethaby, A. (2018). Cochrane corner: long-term hormone therapy for perimenopausal and postmenopausal women. Heart 104, 93-95. doi: 10.1136/heartjnl-2017-311583

Mauvais-Jarvis, F., Manson, J. E., Stevenson, J. C., and Fonseca, V. A. (2017). Menopausal hormone therapy and type 2 diabetes prevention: evidence, mechanisms, and clinical implications. Endocr. Rev. 38, 173-188. doi: 10.1210/ er.2016-1146

Mazure, C. M., and Swendsen, J. (2016). Sex differences in Alzheimer's disease and other dementias. Lancet Neurol. 15, 451-452. doi: 10.1016/S1474-4422(16) 00067-3

McLean, F. H., Grant, C., Morris, A. C., Horgan, G. W., Polanski, A. J., Allan, K., et al. (2018). Rapid and reversible impairment of episodic memory by a high-fat diet in mice. Sci. Rep. 8:11976. doi: 10.1038/s41598-018-30265-4

Merlo, S., Spampinato, S. F., and Sortino, M. A. (2017). Estrogen and Alzheimer's disease: still an attractive topic despite disappointment from early clinical results. Eur. J. Pharmacol. 817, 51-58. doi: 10.1016/j.ejphar.2017.05.059

Misiak, B., Leszek, J., and Kiejna, A. (2012). Metabolic syndrome, mild cognitive impairment and Alzheimer's disease-the emerging role of systemic low-grade inflammation and adiposity. Brain Res. Bull. 89, 144-149. doi: 10.1016/j. brainresbull.2012.08.003

Morrison, J. H., Brinton, R. D., Schmidt, P. J., and Gore, A. C. (2006). Estrogen, menopause, and the aging brain: how basic neuroscience can inform hormone therapy in women. J. Neurosci. 26, 10332-10348. doi: 10.1523/JNEUROSCI. 3369-06.2006

Mosconi, L., Berti, V., Quinn, C., McHugh, P., Petrongolo, G., Varsavsky, I., et al. (2017). Sex differences in Alzheimer risk: brain imaging of endocrine vs chronologic aging. Neurology 89, 1382-1390. doi: 10.1212/WNL. 0000000000004425

Moser, V. A., and Pike, C. J. (2016). Obesity and sex interact in the regulation of Alzheimer's disease. Neurosci. Biobehav. Rev. 67, 102-118. doi: 10.1016/j. neubiorev.2015.08.021

Moser, V. A., and Pike, C. J. (2017). Obesity accelerates alzheimer-related pathology inAPOE4but notAPOE3Mice. Eneuro 4, ENEURO.77-ENEURO.17. doi: 10. 1523/ENEURO.0077-17.2017

Nelson, J. F., Felicio, L. S., Randall, P. K., Sims, C., and Finch, C. E. (1982). A longitudinal study of estrous cyclicity in aging C57BL/6J mice: I. Cycle frequency, length and vaginal cytology. Biol. Reprod. 27, 327-339. doi: 10.1095/ biolreprod27.2.327

O'Brien, P. D., Hinder, L. M., Callaghan, B. C., and Feldman, E. L. (2017). Neurological consequences of obesity. Lancet Neurol. 16, 465-477. doi: 10.1016/ S1474-4422(17)30084-4

Oddo, S., Caccamo, A., Shepherd, J. D., Murphy, M. P., Golde, T. E., Kayed, R., et al. (2003). Triple-transgenic model of Alzheimer's disease with plaques and tangles: intracellular Abeta and synaptic dysfunction. Neuron 39, 409-421. doi: 10.1016/s0896-6273(03)00434-3

Papadakis, G. E., Hans, D., Rodriguez, E. G., Vollenweider, P., Waeber, G., Marques-Vidal, P., et al. (2018). Menopausal hormone therapy is associated with reduced total and visceral adiposity: the osteolaus cohort. J. Clin. Endocrinol. Metab. 103, 1948-1957. doi: 10.1210/jc.2017-02449

Park, H. R., Park, M., Choi, J., Park, K.-Y., Chung, H. Y., and Lee, J. (2010). A high-fat diet impairs neurogenesis: involvement of lipid peroxidation and brain-derived neurotrophic factor. Neurosci. Lett. 482, 235-239. doi: 10.1016/ j.neulet.2010.07.046

Park, S. K., Harlow, S. D., Zheng, H., Karvonen-Gutierrez, C., Thurston, R. C., Ruppert, K., et al. (2017). Association between changes in oestradiol and follicle-stimulating hormone levels during the menopausal transition and risk of diabetes. Diabet. Med. 34, 531-538. doi: 10.1111/dme.13301

Pike, C. J. (2017). Sex and the development of Alzheimer's disease. J. Neurosci. Res. 95, 671-680. doi: 10.1002/jnr.23827

Pike, C. J., Carroll, J. C., Rosario, E. R., and Barron, A. M. (2009). Protective actions of sex steroid hormones in Alzheimer's disease. Front. Neuroendocrinol. 30:239-258. doi: 10.1016/j.yfrne.2009.04.015

Pugazhenthi, S., Qin, L., and Reddy, P. H. (2017). Common neurodegenerative pathways in obesity, diabetes, and Alzheimer's disease. Biochim. Biophys. Acta Mol. Basis Dis. 1863, 1037-1045. doi: 10.1016/j.bbadis.2016.04.017

Purnell, J. Q., Urbanski, H. F., Kievit, P., Roberts, C. T., and Bethea, C. L. (2019). Estradiol replacement timing and obesogenic diet effects on body composition and metabolism in postmenopausal macaques. Endocrinology 160, 899-914. doi: 10.1210/en.2018-00884

Shao, H., Breitner, J. C. S., Whitmer, R. A., Wang, J., Hayden, K., Wengreen, H., et al. (2012). Hormone therapy and Alzheimer disease dementia: new findings from the Cache County Study. Neurology 79, 1846-1852. doi: 10.1212/WNL. ob013e318271f823

Stubbins, R. E., Najjar, K., Holcomb, V. B., Hong, J., and Núñez, N. P. (2012). Oestrogen alters adipocyte biology and protects female mice from adipocyte inflammation and insulin resistance. Diabetes Obes. Metab. 14, 58-66. doi: 10.1111/j.1463-1326.2011.01488.x

Thaler, J. P., Yi, C.-X., Schur, E. A., Guyenet, S. J., Hwang, B. H., Dietrich, M. O., et al. (2012). Obesity is associated with hypothalamic injury in rodents and humans. J. Clin. Invest. 122, 153-162. doi: 10.1172/JCI59660

Vaucher, E., Reymond, I., Najaffe, R., Kar, S., Quirion, R., Miller, M. M., et al. (2002). Estrogen effects on object memory and cholinergic receptors in young and old female mice. Neurobiol. Aging 23, 87-95. doi: 10.1016/s0197-4580(01) 00250-0

Villa, A., Vegeto, E., Poletti, A., and Maggi, A. (2016). Estrogens, neuroinflammation, and neurodegeneration. Endocr. Rev. 37, 372-402. doi: 10.1210/er.2016-1007

Vinters, H. V. (2015). Emerging concepts in Alzheimer's disease. Annu. Rev. Pathol. 10, 291-319. doi: 10.1146/annurev-pathol-020712-163927

Walker, J. M., Dixit, S., Saulsberry, A. C., May, J. M., and Harrison, F. E. (2017). Reversal of high fat diet-induced obesity improves glucose tolerance, inflammatory response, $\beta$-amyloid accumulation and cognitive decline in the APP/PSEN1 mouse model of Alzheimer's disease. Neurobiol. Dis. 100, 87-98. doi: 10.1016/j.nbd.2017.01.004

Webers, A., Heneka, M. T., and Gleeson, P. A. (2019). The role of innate immune responses and neuroinflammation in amyloid accumulation and progression of Alzheimer's disease. Immunol. Cell Biol. 98, 28-41. doi: 10.1111/imcb.12301

Wend, K., Wend, P., and Krum, S. A. (2012). Tissue-specific effects of loss of estrogen during menopause and aging. Front. Endocrinol. (Lausanne) 3:19. doi: 10.3389/fendo.2012.00019

Whitmer, R. A., Quesenberry, C. P., Zhou, J., and Yaffe, K. (2011). Timing of hormone therapy and dementia: the critical window theory revisited. Ann. Neurol. 69, 163-169. doi: 10.1002/ana.22239

Winocur, G., and Greenwood, C. E. (2005). Studies of the effects of high fat diets on cognitive function in a rat model. Neurobiol. Aging 26(Suppl. 1), 46-49. doi: 10.1016/j.neurobiolaging.2005.09.003

Yaffe, K., Sawaya, G., Lieberburg, I., and Grady, D. (1998). Estrogen therapy in postmenopausal women: effects on cognitive function and dementia. JAMA 279, 688-695. doi: 10.1001/jama.279.9.688

Yasrebi, A., Rivera, J. A., Krumm, E. A., Yang, J. A., and Roepke, T. A. (2017). Activation of estrogen response element-independent ER $\alpha$ signaling protects female mice from diet-induced obesity. Endocrinology 158, 319-334. doi: 10. 1210/en.2016-1535

Yeh, C.-W., Liu, H.-K., Lin, L.-C., Liou, K.-T., Huang, Y.-C., Lin, C.H., et al. (2017). Xuefu Zhuyu decoction ameliorates obesity, hepatic steatosis, neuroinflammation, amyloid deposition and cognition impairment 
in metabolically stressed APPswe/PS1dE9 mice. J. Ethnopharmacol. 209, 50-61. doi: 10.1016/j.jep.2017.07.036

Yilmaz, C., Karali, K., Fodelianaki, G., Gravanis, A., Chavakis, T., Charalampopoulos, I., et al. (2019). Neurosteroids as regulators of neuroinflammation. Front. Neuroendocrinol. 55:100788. doi: 10.1016/j.yfrne. 2019.100788

Yin, F., Yao, J., Sancheti, H., Feng, T., Melcangi, R. C., Morgan, T. E., et al. (2015). The perimenopausal aging transition in the female rat brain: decline in bioenergetic systems and synaptic plasticity. Neurobiol. Aging 36, 2282-2295. doi: 10.1016/j.neurobiolaging.2015.03.013

Yun, J., Yeo, I. J., Hwang, C. J., Choi, D.-Y., Im, H.-S., Kim, J. Y., et al. (2018). Estrogen deficiency exacerbates $\mathrm{A} \beta$-induced memory impairment through enhancement of neuroinflammation, amyloidogenesis and NF-кB activation in ovariectomized mice. Brain Behav. Immun. 73, 282-293. doi: 10.1016/j.bbi. 2018.05.013

Conflict of Interest: The authors declare that the research was conducted in the absence of any commercial or financial relationships that could be construed as a potential conflict of interest.

Copyright $(2020$ Christensen, Liu and Pike. This is an open-access article distributed under the terms of the Creative Commons Attribution License (CC BY). The use, distribution or reproduction in other forums is permitted, provided the original author(s) and the copyright owner(s) are credited and that the original publication in this journal is cited, in accordance with accepted academic practice. No use, distribution or reproduction is permitted which does not comply with these terms. 